\title{
A Fast Alternating Time-Splitting Approach for Learning Partial Differential Equations
}

\author{
- Revision Summary and Replies to Review Comments
}

Zhenyu Zhao, Zhouchen Lin, Yi Wu and Jianlong Wu

First of all, the authors would like to thank the reviewers for bringing up valuable questions/suggestions to improve our paper. We have tried our best to revise the manuscript. All the comments have been addressed appropriately.

In the following, we first provide a "Revision Summary" in Section 1 to list the difference between the original manuscript and the revised one, so that the reviewers and the editor can easily identify what changes we have made. We further provide detailed replies to the review comments in Section 2.

\section{Revision Summary}

The differences between the original manuscript and the revised version are summarized as follows.

(C) 2016. This manuscript version is made available under the Elsevier user license http://www.elsevier.com/open-access/userlicense/1.0/ 
1. Following the suggestions from Reviewer \#1, we have gone through the paper more carefully to correct some linguistic mistakes and tried our best to make the paper read smoothly. We also add a flowchart and a graphical abstract of the proposed approach.

2. Following the suggestions from Reviewers \#2, we add Subsection 4.1 to discuss the complexity for Algorithms 1 and 2. Besides, we have made more detailed comparison between the proposed FATSA and GDM to prove that FATSA outperforms GDM in different aspects. Moreover, we add the experiments on noisy color image demosaicking in Subsection 5.4.2. We choose the challenging Image Denoising Benchmark database proposed by Estrada et al. to further evaluate the performance of the proposed FATSA.

3. All the typos and other minor comments from reviewers have been properly addressed in the new version. All the suggested references were cited appropriately.

\section{Detailed Replies to Review Comments}

The replies below are ordered as the questions appearing in the comments.

\subsection{To Reviewer \#1:}

1. English level of the manuscript should be updated by native English speaker.

Reply: We have examined the manuscript carefully to find and correct typographical errors and mistakes in grammar, style, and spelling. We try our best to make sure that every sentence can be read smoothly and hope it could meet the 
standards of Neurocomputing.

2. Box-chart of the proposed approach should be added.

Reply: We are not sure about the exact meaning of "Box-chart". To the best of our knowledge, the two closest words are "Box plot" and "flowchart". According to Wikipedia, a box plot is a convenient way of graphically depicting groups of numerical data through their quartiles in descriptive statistics. In our paper, we do not depict groups of numerical data. So we think that giving a flowchart of the proposed approach is suitable. Accordingly, we have added a flowchart (see Figure 1) of the proposed approach in Section 3.1.

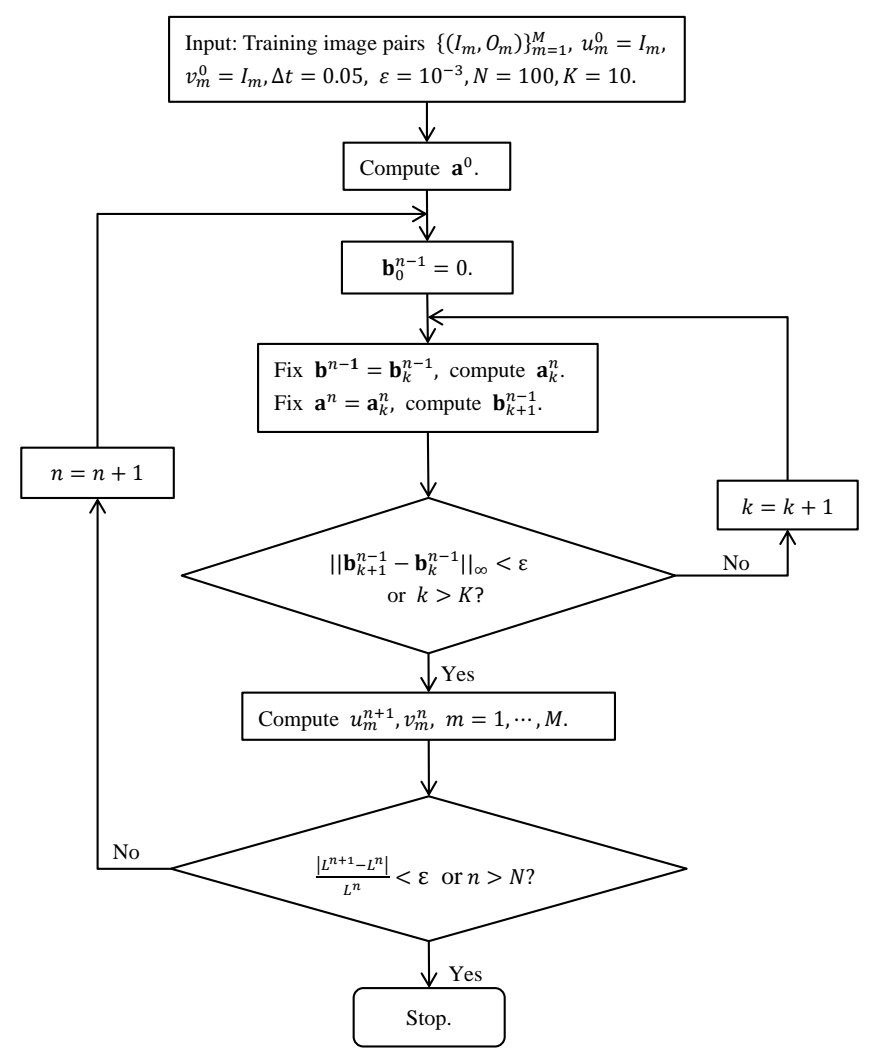

Figure 1: Flowchart of the proposed FATSA. 


\section{Graphical abstract should be provided in straightforward manner.}

Reply: We have asked the editor about the issue of graphical abstract. He/She replied that there is no submission term "Graphical Abstracts" from the dropdown menu in Neurocomputing submission system. Following his/her suggestion, we put the graphical abstract in the main manuscript file. We also present it here. Unlike GDM, we minimize the difference between the expected output and the actual output of PDEs at each time step. This makes the learning procedure much faster, simpler and more flexible.

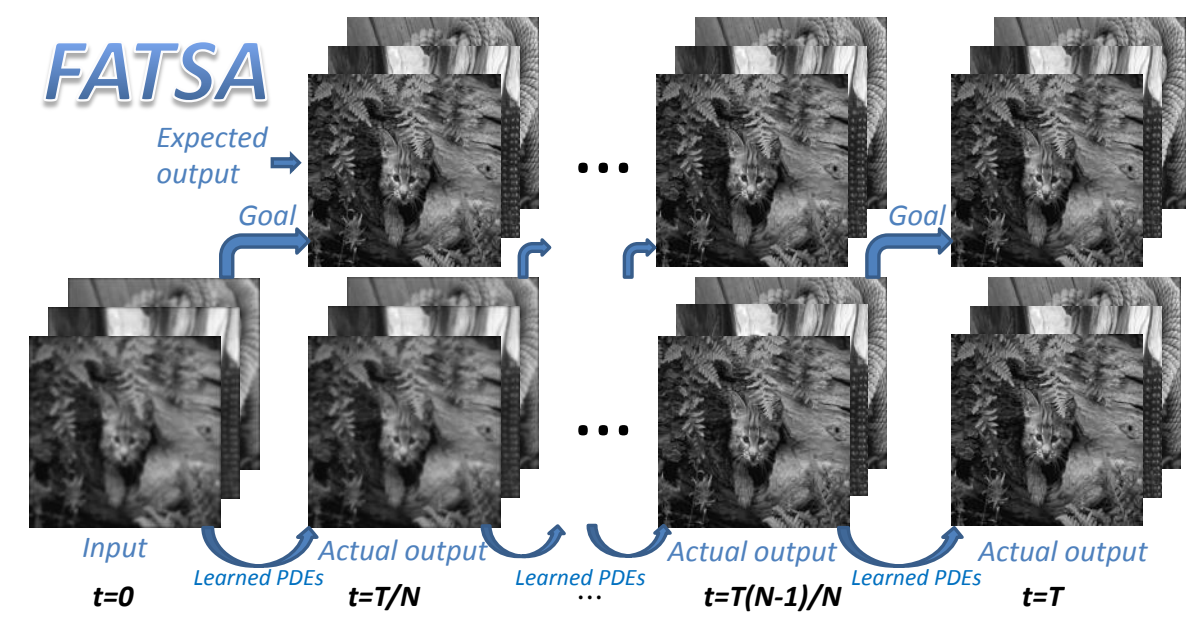

Graphical Abstract: FATSA tries to minimize the difference between the expected output and the actual output of PDEs at each time step to learn the coefficients of PDEs.

4. Introduction and literature review section should be updated by adding relevant citations specially from Neurocomputing journal.

Reply: We have cited four additional papers from Neurocomputing journal in the revised version of the paper (i.e., $[5,7,10]$ in the first paragraph of Section 1 and [33] in the second paragraph of Section 6). These papers applied the traditional PDEs to image denoising, enhancement and tracking. 


\subsection{To Reviewer \#2:}

1. In the introduction, the author briefly introduce some existing methods about PDE. I would recommend to add one relevant paper which introduced low level optical flow estimation using Markov model and Bayesian estimation for latent variables for different human actions. Q. Cai, Y. Yin, H. Man, DSPM: Dynamic Structure Preserving Map for Action Recognition, ICME, 2013.

Reply: We have added the reference in the revised version of the paper as [12] (please see the first paragraph of Section 1 and the second paragraph of Section 6). It introduced low level optical flow estimation for high level human action recognition.

2. The author claims 3 major contributions as fast, flexible, simple. But I did not find the evidence or results to support these advantages.

Reply: We have rewritten Subsection 4.2 (comparison between FATSA and GDM) to explain and emphasize the contributions we make. For the training speed, we compare the computation time between FATSA and GDM in (Subsection 5.5). Experiments demonstrate that FATSA can greatly reduce the training time as well as the training error. For the simplicity, FATSA avoids to compute the adjoint PDEs for evaluating the Gâteaux derivatives of the objective functional. For the flexibility, FATSA can also work when more general regularizations (e.g., non-smooth regularization) and extra constraints on the linear combination coefficients are added.

3. What is the complexity for algorithm 1 and 2? Please make more detailed discussion about this issue. 
Reply: We have added a subsection (Subsection 4.1) to discuss the complexity of Algorithms 1 and 2. We quote it below.

As we set the maximum time $T=N \Delta t$, we can compute an asymptotic worst-case bound for the time complexity. We denote $n_{\mathbf{i n v}}$ as the number of invariants (for gray image $n_{\mathbf{i n v}}=17$, for color image $n_{\mathbf{i n v}}=69$ ) and $|\Omega|$ as the number of pixels in $\Omega$. Problems (13) and (19) are convex quadratic optimization problems for the positive definite $\mathbf{G}_{1}$ and $\mathbf{G}_{2}$, respectively. The cubic time complexity $O\left(n_{\text {inv }}^{3}\right)$ is an asymptotic worst-case bound. For each iteration in Algorithm 2, we need to update $v_{m}^{n}$. So the time complexity is $O(|\Omega|)$. When we have $M$ image training pairs and the maximum number of iteration is $K$, $O\left(K\left(M|\Omega|+n_{\text {inv }}^{3}\right)\right)$ is the worst-case bound for Algorithm 2. As the terminal time $T=N \Delta t$, the worst-case bound for Algorithm 1 is $O\left(K N\left(M|\Omega|+n_{\text {inv }}^{3}\right)\right)$.

4. The datasets used in the experiment are simple and not new. I suggest to use more complex image datasets that can verify the robustness of the algorithm.

Reply: It is very important to note that we aim to propose a simpler and faster approach for learning PDEs instead of proposing a new model to handle various image processing problems. We have highlighted this in the first paragraph of Section 5. So the choice of datasets is actually non-critical. The datasets used in this paper are usually used to evaluate the performance of PDEs-based methods. For image deblurring, several datasets consist of images with large blur, which the PDEs-based methods cannot handle. Therefore, we validate the performance of FATSA for this task based on the blurred version of the Berkeley image database. For image denoising, we use the dataset proposed by Liu et al. to test the performance of the proposed FATSA. This dataset consists of images with unknown natural noises, which is very challenging rather than being "sim- 
ple." For image demosaicking, the most widely used database is the Kodak image database. Almost all the demosaicking methods are tested on this database. So we also choose it to test the performance of FATSA. However, all the images of this database are noiseless. So we add new experiments on the Image Denoising Benchmark database for noisy color image demosaicking. As Ehrhardt et al. (reference [18] of the paper, published in IEEE TIP 2014) have done the demosaicking experiments on this database, we also choose it to test the proposed FATSA. The results are shown in Subsection 5.4.2. The running time is also added in Subsection 5.5. 


\title{
A Fast Alternating Time-Splitting Approach for Learning Partial Differential Equations
}

\author{
Zhenyu Zhao ${ }^{\mathrm{a}}$, Zhouchen $\operatorname{Lin}^{\mathrm{b}, *}, \mathrm{Yi} \mathrm{Wu}^{\mathrm{a}}$ \\ ${ }^{a}$ Department of Mathematics, School of Science, \\ National University of Defense Technology, P. R. China \\ ${ }^{b}$ Key Lab. of Machine Perception (MOE), School of EECS, \\ Peking University, P. R. China
}

\begin{abstract}
Learning-based partial differential equations (PDEs), which combine fundamental differential invariants into a nonlinear regressor, have been successfully applied to several computer vision and image processing problems. However, the gradient descent method (GDM) for solving the linear combination coefficients among differential invariants is time-consuming. Moreover, when the regularization or constraints on the coefficients become more complex, it is troublesome or even impossible to deduce the gradients. In this paper, we propose a new algorithm, called fast alternating time-splitting approach (FATSA), to solve the linear combination coefficients. By minimizing the difference between the expected output and the actual output of PDEs at each time step, FATSA can solve the linear combination coefficients much faster than GDM. More complex regularization or constraints can also be easily incorporated. Extensive experiments demonstrate that our proposed FATSA outperform GDM in both speed and quality.

Keywords: Learning-Based PDEs, PDE constrained optimal control, FATSA, Computer vision, Image processing
\end{abstract}

\section{Introduction}

Partial differential equations (PDEs) have been successfully used to solve many practical problems in computer vision and image processing $[1,2,3]$, such as denoising $[4,5]$, enhancement [6, 7], inpainting [8], segmentation [9, 10], and optical flow computation $[11,12]$. However, It is usually difficult to design a PDE system for a particular task which requires high mathematical skills and good insight into the problem. According to [13], the existing methods of designing PDEs can be mainly classified into two groups. The methods of the first group write down PDEs directly, which requires good

\footnotetext{
* Corresponding author.

Email addresses: dwightzzy@gmail.com (Zhenyu Zhao), zlin@pku.edu.cn (Zhouchen Lin), wuyi_work@sina.com (Yi Wu)
} 
mathematical understandings on the properties of the PDEs. The methods of second group first define an energy functional [14], which pursues the expected properties of the output image or video, and then derive evolution equations by computing the Euler-Lagrange variation of the energy functional. For example, the ROF model [15] and TV- $L_{1}[16]$ for image denoising are designed directly, while the Nambu model [17] and the PL model [18] for color image processing are designed in the variational way.

To reduce the difficulty in designing PDEs for complex vision problems, Liu and Lin et al. [13] proposed a framework that learns PDEs from training image pairs recently. They first considered learning PDEs for grayscale image restoration [19], which involve an anisotropic diffusion term. Then they generalized the idea significantly by linearly combining fundamental differential invariants that are invariant to translation and rotation. These differential invariants serve as "bases" of differential operators $[13,20]$. They utilized the gradient descent method (GDM) to solve the linear combination coefficients. The learnt PDEs have been successfully applied to various problems, such as image denoising, debluring, object detection, color to gray and demosaicking [13, 21, 22].

However, GDM has several drawbacks. First, the convergence speed of GDM is very slow due to the fact that objective functional is flat. Experiments show that the magnitude of gradient is usually at the order of $10^{-3}$ (Fig. 4), even at the beginning iterations. Therefore, the solution of GDM does not improve the initial value very much. Second, it needs to solve the adjoint PDEs to obtain the gradient, which is difficult to deduce and also time-consuming. Third, when the regularization or constraints on the linear combination coefficients become more complex, e.g., we use $L_{1}$ norm as the regularizer or add boundedness constraints, the deduction of gradient becomes very involved or even non-existent because of the non-differentiablity of the objective functional. Last, the quality of learnt PDEs is not very good. For example, the magnitudes of coefficients are unbalanced. We can see from Fig. 4(a) that most of $a_{i}(t)$ 's are close to zeros while some jump to more than 20. This can cause numerical instability as the differential invariants involve multiplications of second order derivatives. As a result, blowup might occur when we apply the learnt PDEs to test images. Moreover, we can also see from Fig. 4(c) that $b_{i}(t)$ 's are very close to zeros, which means that the indicator function is actually ineffective.

To overcome the above short-comings of GDM, we propose a new method, called fast alternating time-splitting approach (FATSA), to solve the linear combination coefficients. We first discretize the PDEs in time. Then we minimize the difference between the expected output (ground truth) and the actual output of the PDEs at each time step $n$, which is a nonlinear regression problem and can be 
solved by alternately minimizing $a_{i}(n \Delta t)$ 's and $b_{j}((n-1) \Delta t)$ 's. In such a greedy manner, the linear combination coefficients can be updated sequentially in time. Based on FATSA, it is convenient to add constrains and regularization on the coefficients, even when these constrains and regularization are non-differentiable. Moreover, we do not need to deduce and compute the adjoint PDEs any longer. Besides, compared with GDM, FATSA can greatly reduce the training time and the training error. For grayscale images, the speed of training is accelerated by ten times. For color images, the training time is cut by half. In summary, the contributions of this paper are summarized as follows:

- We propose a new fast alternating time-splitting approach (FATSA) to solve the PDE constrained optimal control problem, which not only speeds up the learning process, but also improves the results.

- Compared with GDM, FATSA is much simpler. It computes the linear combination coefficients in temporal order. It avoids to compute the adjoint PDEs for evaluating the Gâteaux derivatives [23] of the objective functional.

- FATSA is much more flexible than GDM. When we add more general regularizations (e.g., nonsmooth regularization) and extra constraints on the linear combination coefficients, it can also improve the results.

The rest of the paper is organized as follows. First of all, we review the learning-based PDEs methods briefly in Section 2. Then we present the main idea of FATSA and the details of alternating minimization in Section 3. In Section 4, we discuss the complexity of FATSA and make a detailed comparison with GDM [13]. We also extend FATSA to solve learning-based PDEs for vector-valued image processing problems. Then in Section 5, we compare the performance of FATSA and GDM on some computer vision and image processing problems. Finally, we conclude our paper and discuss the future work in Section 6.

\section{Learning-based PDEs}

In this section, we briefly review the framework of learning-based PDEs for computer vision and image processing problems. More details can be found in [13, 21, 22].

\subsection{Mathematical formulation}

The current learning-based PDEs are grounded on the translational and rotational invariance of computer vision and image processing problems. Namely, when the input image is translated or 
Table 1: Fundamental differential invariants up to the second order, where $t r$ is the trace operator and $\nabla f$ and $\mathbf{H}_{f}$ are the gradient and the Hessian matrix of function $f$, respectively.

\begin{tabular}{ll}
$j$ & $\operatorname{inv}_{j}(u, v)$ \\
\hline $0,1,2$ & $1, v, u$ \\
3,4 & $\|\nabla v\|^{2}=v_{x}^{2}+v_{y}^{2},\|\nabla u\|^{2}=u_{x}^{2}+u_{y}^{2}$ \\
5 & $(\nabla v)^{T} \cdot \nabla u=v_{x} u_{x}+v_{y} u_{y}$ \\
6,7 & $\operatorname{tr}\left(\mathbf{H}_{v}\right)=v_{x x}+v_{y y}, \operatorname{tr}\left(\mathbf{H}_{u}\right)=u_{x x}+u_{y y}$ \\
8 & $(\nabla v)^{T} \cdot \mathbf{H}_{v} \cdot \nabla v=v_{x}^{2} v_{x x}+2 v_{x} v_{y} v_{x y}+v_{y}^{2} v_{y y}$ \\
9 & $(\nabla v)^{T} \cdot \mathbf{H}_{u} \cdot \nabla v=v_{x}^{2} u_{x x}+2 v_{x} v_{y} u_{x y}+v_{y}^{2} u_{y y}$ \\
10 & $(\nabla v)^{T} \cdot \mathbf{H}_{v} \cdot \nabla u=v_{x} u_{x} v_{x x}+\left(v_{x} u_{y}+u_{x} v_{y}\right) v_{x y}+v_{y} u_{y} v_{y y}$ \\
11 & $(\nabla v)^{T} \cdot \mathbf{H}_{u} \cdot \nabla u=v_{x} u_{x} u_{x x}+\left(v_{x} u_{y}+u_{x} v_{y}\right) u_{x y}+v_{y} u_{y} u_{y y}$ \\
12 & $(\nabla u)^{T} \cdot \mathbf{H}_{v} \cdot \nabla u=u_{x}^{2} v_{x x}+2 u_{x} u_{y} v_{x y}+u_{y}^{2} v_{y y}$ \\
13 & $(\nabla u)^{T} \cdot \mathbf{H}_{u} \cdot \nabla u=u_{x}^{2} u_{x x}+2 u_{x} u_{y} u_{x y}+u_{y}^{2} u_{y y}$ \\
14 & $\operatorname{tr}\left(\mathbf{H}_{v}^{2}\right)=v_{x x}^{2}+2 v_{x y}^{2}+v_{y y}^{2}$ \\
15 & $\operatorname{tr}\left(\mathbf{H}_{v} \cdot \mathbf{H}_{u}\right)=v_{x x} u_{x x}+2 v_{x y} u_{x y}+v_{y y} u_{y y}$ \\
16 & $\operatorname{tr}\left(\mathbf{H}_{u}^{2}\right)=u_{x x}^{2}+2 u_{x y}^{2}+u_{y y}^{2}$ \\
\hline
\end{tabular}

rotated, the output image should be translated or rotated accordingly. Then it can be proven that the governing equations are functions of fundamental differential invariants, which form "bases" of all differential invariants that are invariant with respect to translation and rotation. We assume that the evolution of the image $u$ is guided by an indicator function $v$, which collects large scale information. As shown in Table 1, there are 17 fundamental differential invariants $\left\{\operatorname{inv}_{i}(u, v), i=0, \cdots, 16\right\}$ up to the second order. For brevity, we denote $\operatorname{inv}(u, v)=\left[\operatorname{inv}_{0}(u, v), \operatorname{inv}_{1}(u, v), \cdots, \operatorname{inv}_{16}(u, v)\right]^{T}$, where $(\cdot)^{T}$ denoted the transpose of matrix (or vector).

The simplest function of fundamental differential invariants is a linear combination of them. Therefore, learning the PDEs can be transformed into learning the linear combination coefficients among the fundamental differential invariants, which are functions of time $t$ only and independent of spatial variables $[13,21,22]$. To this end, one may prepare a number of input/output training image pairs. By minimizing the difference between the output of PDEs and the ground truth. We set the initial 
function as the input image. This results in a PDEs constrained optimal control problem:

$$
\begin{aligned}
& \min _{\mathbf{a}, \mathbf{b}} E(\mathbf{a}(t), \mathbf{b}(t))=\frac{1}{2} \sum_{m=1}^{M} \int_{\Omega}\left(O_{m}-u_{m}(x, y, T)\right)^{2} \mathrm{~d} \Omega \\
& \quad+\lambda_{1} \sum_{i=0}^{16} \int_{0}^{T} a_{i}^{2}(t) \mathrm{d} t+\lambda_{2} \sum_{i=0}^{16} \int_{0}^{T} b_{i}^{2}(t) \mathrm{d} t, \\
& \text { s.t. } \begin{cases}\frac{\partial u_{m}}{\partial t}-\mathbf{i n v}^{T}\left(u_{m}, v_{m}\right) \cdot \mathbf{a}(t)=0, & (x, y, t) \in Q, \\
u_{m}(x, y, t)=0, & (x, y, t) \in \Gamma, \\
u_{m}(x, y, 0)=I_{m}, & (x, y) \in \Omega, \\
\frac{\partial v_{m}}{\partial t}-\mathbf{i n v}^{T}\left(v_{m}, u_{m}\right) \cdot \mathbf{b}(t)=0, & (x, y, t) \in Q, \\
v_{m}(x, y, t)=0, & (x, y, t) \in \Gamma, \\
v_{m}(x, y, 0)=I_{m}, & (x, y) \in \Omega,\end{cases}
\end{aligned}
$$

where $\left\{\left(I_{m}, O_{m}\right), m=1, \cdots, M\right\}$ denote the $M$ input/output training image pairs, $u_{m}(x, y, t)$ is the evolution image at time $t$ with respect to the input image $I_{m}, v_{m}(x, y, t)$ is the corresponding indicator function, $\Omega \subset \mathbb{R}^{2}$ is the (rectangular) region occupied by the image ${ }^{1}, T$ is the temporal span of evolution which can be normalized as $1, Q=\Omega \times[0, T], \Gamma=\partial \Omega \times[0, T]$, and $\partial \Omega$ denotes the boundary of $\Omega$. The last two terms in (1) are regularization terms on the coefficients $a_{i}(t)$ and $b_{i}(t)$. We denote it as $\mathbf{a}(t)=\left[a_{0}(t), a_{1}(t), \cdots, a_{16}(t)\right]^{T}$ and $\mathbf{b}(t)=\left[b_{0}(t), b_{1}(t), \cdots, b_{16}(t)\right]^{T}$ for brevity. $\operatorname{For}_{\operatorname{inv}_{i}}(v, u)$, it can be acquired by simply switching $u$ and $v$ in $\operatorname{inv}_{i}(u, v)$.

\subsection{Gradient descend method}

Liu and Lin et al. [13, 21, 22] proposed a gradient descend method (GDM) to solve the optimal coefficients $a_{i}(t)$ and $b_{i}(t)$, where the "gradient" is actually the Gâteaux derivatives [23] of the objective functional $E$ with respect to the coefficient functions. The Gâteaux derivatives of $E$ with respect to $a_{i}(t)$ and $b_{i}(t)$ are as follows:

$$
\left\{\begin{array}{l}
\frac{D E}{D a_{i}}=\lambda_{1} a_{i}-\sum_{m=1}^{M} \int_{\Omega} \varphi_{m} \operatorname{inv}_{i}\left(u_{m}, v_{m}\right) \mathrm{d} \Omega \\
\frac{D E}{D b_{i}}=\lambda_{2} b_{i}-\sum_{m=1}^{M} \int_{\Omega} \psi_{m} \operatorname{inv}_{i}\left(v_{m}, u_{m}\right) \mathrm{d} \Omega
\end{array}\right.
$$

where $\varphi$ and $\psi$ are the solutions to the adjoint equations. Since the adjoint equations are very complex, we omit the details here.

Based on the Gâteaux derivatives, the local optimal solutions of $\mathbf{a}(t)$ and $\mathbf{b}(t)$ can be computed via linear searching along descent directions (e.g., conjugate gradient).

\footnotetext{
${ }^{1}$ The images are padded with zeros of several pixels width around them, so that the Dirichlet boundary conditions, $u_{m}(x, y, t)=0, v_{m}(x, y, t)=0,(x, y, t) \in \Gamma$, are naturally fulfilled.
} 


\section{FATSA: fast alternating time-splitting approach}

The Gâteaux derivatives shown above are only available for smooth regularizations, such as squared $L_{2}$ norms in (1), on the coefficients. However, when the regularizations are non-smooth or extra constraints are added to the coefficients, it is nearly impossible to deduce the Gâteaux derivatives. Besides, it is time-consuming to solve the adjoint equations. What's more, as the objective functional is quite flat, the magnitudes of all the Gâteaux derivatives are very small. As a result, the speed of GDM is very slow and the obtained coefficients are not very far from their initial values. Consequently, the performance of learnt PDEs is not always satisfactory.

To tackle the above drawbacks of GDM, in this paper we propose a greedy strategy that minimizes the difference between the expected outputs (ground truth) and the actual outputs of the PDEs at every time step, rather than minimizing the difference between the expected outputs and the actual outputs of the PDEs at $T$ only. In view of the highly non-convex nature of the PDE constrained optimal control problem, it is very easy for GDM to be stuck at local minima during solving the optimal coefficients. Compared with GDM, our new greedy strategy is more effective, which can be demonstrated by experiments.

In the proposed approach, we first discretize the temporal variable $t$ with a step size $\Delta t$ and denote it as $t_{i}=i \cdot \Delta t, i=0, \cdots, N$. At each time step $t_{n+1}$, we minimize

$$
L\left(t_{n+1}\right)=\frac{1}{2} \sum_{m=1}^{M} \int_{\Omega}\left(O_{m}-u_{m}\left(x, y, t_{n+1}\right)\right)^{2} \mathrm{~d} \Omega,
$$

where $u_{m}\left(x, y, t_{n+1}\right)$ is the solution of PDEs (2) at time $t_{n+1}$. The motivation of minimizing (4) is to attract $u_{m}$ to the desired output $O_{m}$ as fast as possible, while GDM minimizes

$$
E^{\prime}=\frac{1}{2} \sum_{m=1}^{M} \int_{\Omega}\left(O_{m}-u_{m}(x, y, T)\right)^{2} \mathrm{~d} \Omega,
$$

which is the difference between the expected output and the final outputs of the PDEs $(t=T)$.

In the sequel, we simply use $u_{m}^{n}$ to denote $u_{m}\left(x, y, t_{n}\right)$. Other notations, such as $v_{m}^{n}, \mathbf{a}^{n}, \mathbf{b}^{n}$ and $L^{n}$, are denoted in the same way.

We use the following forward scheme to approximate the governing equations in (2),

$$
\begin{cases}u_{m}^{n+1}=u_{m}^{n}+\Delta t \cdot \mathbf{i n v}^{T}\left(u_{m}^{n}, v_{m}^{n}\right) \cdot \mathbf{a}^{n}, & n \geq 0 \\ v_{m}^{n}=v_{m}^{n-1}+\Delta t \cdot \mathbf{i n v}^{T}\left(v_{m}^{n-1}, u_{m}^{n-1}\right) \cdot \mathbf{b}^{n-1}, & n \geq 1 .\end{cases}
$$

By combining (4) and (6), we can obtain that

$$
L^{n+1}=\frac{1}{2} \sum_{m=1}^{M} \int_{\Omega}\left[O_{m}-u_{m}^{n}-\Delta t \cdot \mathbf{i n v}^{T}\left(u_{m}^{n}, v_{m}^{n}\right) \cdot \mathbf{a}^{n}\right]^{2} \mathrm{~d} \Omega .
$$




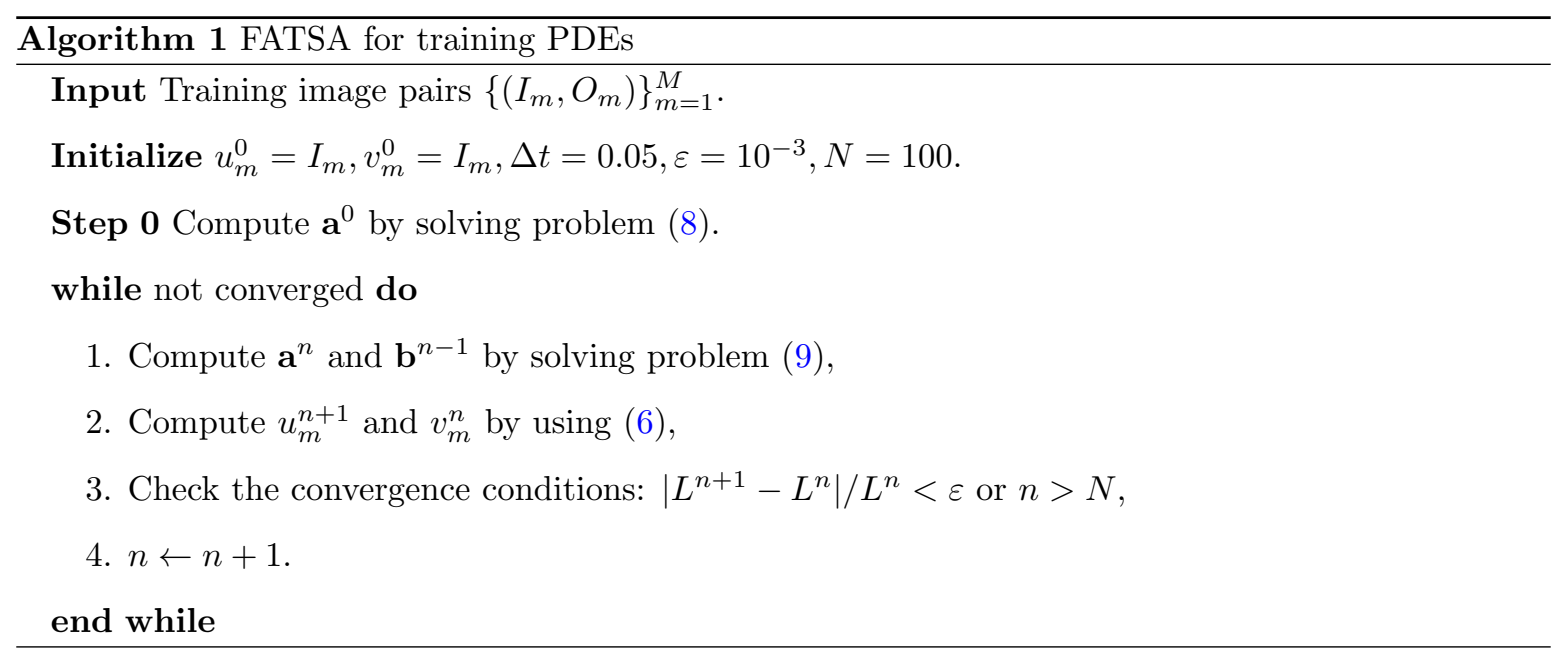

Note that when $n \geq 1, L^{n+1}$ is dependent on $\mathbf{a}^{n}$ and $\mathbf{b}^{n-1}$, where the dependence on $\mathbf{b}^{n-1}$ is due to $v_{m}^{n}$ in $\operatorname{inv}^{T}\left(u_{m}^{n}, v_{m}^{n}\right)$, which can be computed by the second equation of $(6)$. When $n=0, L^{1}$ is only dependent on $\mathbf{a}^{0}$ as $v_{m}^{0}$ is known. So we can initialize $\mathbf{a}^{0}$ by minimizing $L^{1}$. Then $\mathbf{a}^{n}$ and $\mathbf{b}^{n-1}(n \geq 1)$ can be computed sequentially by minimizing $L^{n+1}(n \geq 1)$.

In order to show the flexibility of our new optimization method as well as ensure the computation stability, we further add boundedness constraints on $\mathbf{a}^{n}$ and $\mathbf{b}^{n}$. Then the problem of computing $\mathbf{a}^{0}$ is transformed into

$$
\min _{\mathbf{a}^{0}} L^{1}=\frac{1}{2} \sum_{m=1}^{M} \int_{\Omega}\left[O_{m}-u_{m}^{0}-\Delta t \cdot \mathbf{i n v}^{T}\left(u_{m}^{0}, v_{m}^{0}\right) \cdot \mathbf{a}^{0}\right]^{2} \mathrm{~d} \Omega, \quad \text { s.t. } \quad\left\|\mathbf{a}^{0}\right\|_{\infty} \leq \eta_{1},
$$

and the problem of solving $\mathbf{a}^{n}$ and $\mathbf{b}^{n-1}(n \geq 1)$ is transformed into

$$
\begin{aligned}
\min _{\mathbf{a}^{n}, \mathbf{b}^{n-1}} L^{n+1}= & \frac{1}{2} \sum_{m=1}^{M} \int_{\Omega}\left[O_{m}-u_{m}^{n}-\Delta t \cdot \mathbf{i n v}^{T}\left(u_{m}^{n}, v_{m}^{n}\right)\right]^{2} \mathrm{~d} \Omega, \\
& \text { s.t. }\left\|\mathbf{a}^{n}\right\|_{\infty} \leq \eta_{1},\left\|\mathbf{b}^{n-1}\right\|_{\infty} \leq \eta_{2} .
\end{aligned}
$$

Problem (8) is simply a quadratic optimization problem with a boundedness constraint. As for problem (9), we can use the block coordinate descent method to update $\mathbf{a}^{n}$ and $\mathbf{b}^{n-1}$ alternately. Hence we call our new optimization method as fast alternating time-splitting approach (FATSA), which is summarized in Algorithm 1. We will present the details in the following subsections.

\subsection{Alternating minimization}

In this subsection, we focus on solving (9) by alternating minimization. As for (8), it is easy to see that this problem is same as the one when we update $\mathbf{a}^{n}$ with fixed $\mathbf{b}^{n-1}$ in (9) (see (10) below). So we skip the details of solving (8). 
By alternating minimization, (9) reduces to the following two subproblems. While $\mathbf{b}^{n-1}$ is fixed, we can get $v_{m}^{n}=v_{m}^{n-1}+\Delta t \cdot \mathbf{i n v}^{T}\left(v_{m}^{n-1}, u_{m}^{n-1}\right) \cdot \mathbf{b}^{n-1}$ and $\mathbf{i n v}\left(u_{m}^{n}, v_{m}^{n}\right)$. Then the problem for updating $\mathbf{a}^{n}$ is transformed into

$$
\min _{\mathbf{a}^{n}} L_{a}^{n+1}=\frac{1}{2} \sum_{m=1}^{M} \int_{\Omega}\left[O_{m}-u_{m}^{n}-\Delta t \cdot \mathbf{i n v}^{T}\left(u_{m}^{n}, v_{m}^{n}\right) \cdot \mathbf{a}^{n}\right]^{2} \mathrm{~d} \Omega, \text { s.t. }\left\|\mathbf{a}^{n}\right\|_{\infty} \leq \eta_{1} .
$$

When $\mathbf{a}^{n}$ is fixed, problem (9) is simplified into the following problem

$$
\begin{aligned}
\min _{\mathbf{b}^{n-1}} L_{b}^{n+1}= & \frac{1}{2} \sum_{m=1}^{M} \int_{\Omega}\left[O_{m}-u_{m}^{n}-\Delta t \cdot \mathbf{i n v}^{T}\left(u_{m}^{n}, v_{m}^{n-1}+\Delta t \cdot \mathbf{i n v}^{T}\left(v_{m}^{n-1}, u_{m}^{n-1}\right) \cdot \mathbf{b}^{n-1}\right) \cdot \mathbf{a}^{n}\right]^{2} \mathrm{~d} \Omega, \\
& \text { s.t. }\left\|\mathbf{b}^{n-1}\right\|_{\infty} \leq \eta_{2} .
\end{aligned}
$$

We initialize $\mathbf{b}^{n-1}$ as $\mathbf{0}$ and summarize the process of solving problem (9) in Algorithm 2. The flowchart to solve the whole problem is presented in Fig. 1. In the following subsections, we present how to solve (10) and (11).

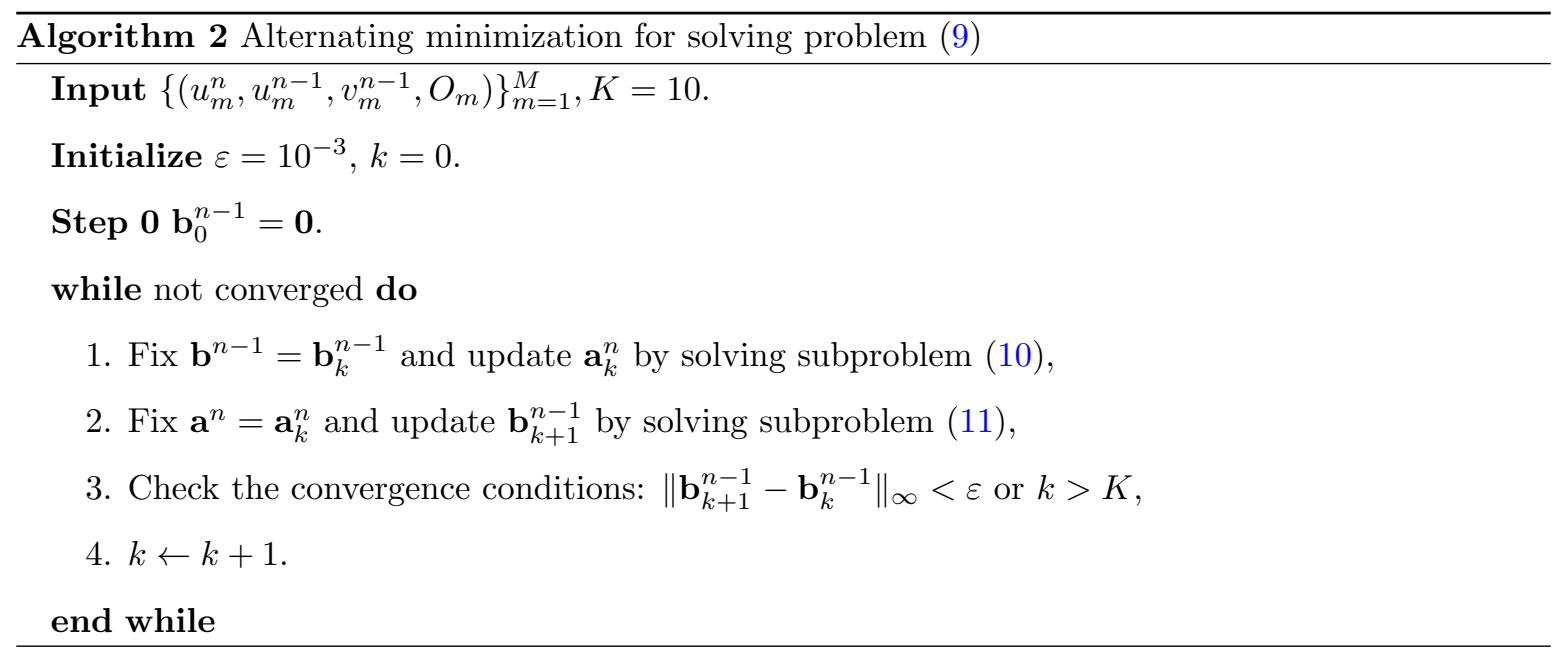

\subsubsection{Solving subproblem (10)}

In this subsection, we present the details of solving subproblem (10). From (10),

$$
\begin{aligned}
L_{a}^{n+1}= & \frac{1}{2} \sum_{m=1}^{M} \int_{\Omega}\left[O_{m}-u_{m}^{n}-\Delta t \cdot \mathbf{i n v}^{T}\left(u_{m}^{n}, v_{m}^{n}\right) \cdot \mathbf{a}^{n}\right]^{2} \mathrm{~d} \Omega \\
= & \frac{1}{2} \sum_{m=1}^{M} \int_{\Omega}(\Delta t)^{2} \cdot\left(\mathbf{a}^{n}\right)^{T} \cdot \mathbf{i n v}\left(u_{m}^{n}, v_{m}^{n}\right) \cdot \mathbf{i n v}^{T}\left(u_{m}^{n}, v_{m}^{n}\right) \cdot \mathbf{a}^{n} \mathrm{~d} \Omega \\
& -\sum_{m=1}^{M} \int_{\Omega} \Delta t \cdot\left(O_{m}-u_{m}^{n}\right) \cdot \mathbf{i n v}^{T}\left(u_{m}^{n}, v_{m}^{n}\right) \cdot \mathbf{a}^{n} \mathrm{~d} \Omega+\frac{1}{2} \sum_{m=1}^{M} \int_{\Omega}\left(O_{m}-u_{m}^{n}\right)^{2} \mathrm{~d} \Omega .
\end{aligned}
$$




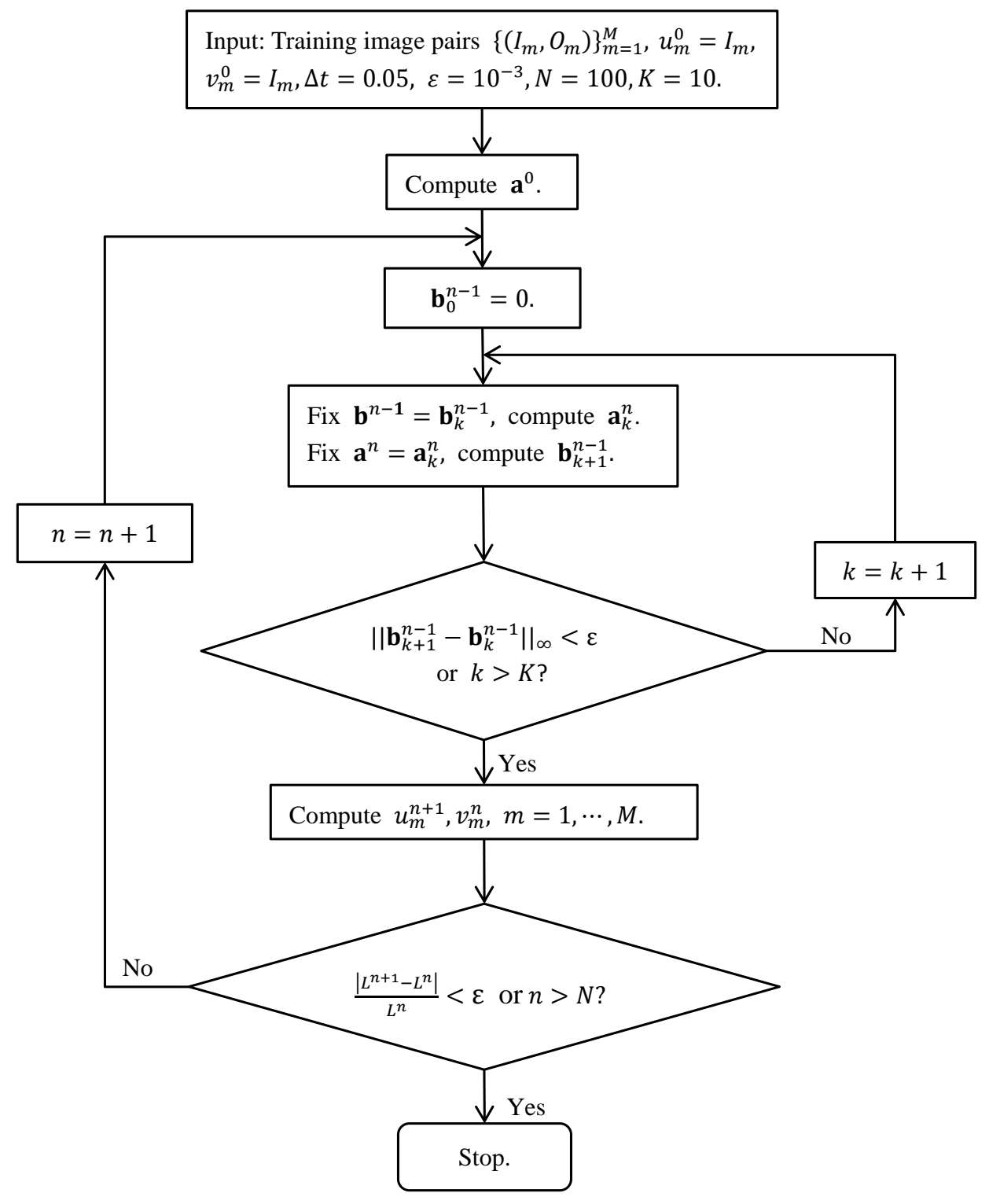

Figure 1: Flowchart of the proposed FATSA.

Note that $\mathbf{a}^{n}$ is independent of $(x, y)$. Denote

$$
\left\{\begin{array}{l}
\mathbf{g}_{1}=\Delta t \cdot \sum_{m=1}^{M} \int_{\Omega}\left(O_{m}-u_{m}^{n}\right) \cdot \operatorname{inv}\left(u_{m}^{n}, v_{m}^{n}\right) \mathrm{d} \Omega \\
\mathbf{G}_{1}=(\Delta t)^{2} \cdot \sum_{m=1}^{M} \int_{\Omega} \operatorname{inv}\left(u_{m}^{n}, v_{m}^{n}\right) \cdot \mathbf{i n v}^{T}\left(u_{m}^{n}, v_{m}^{n}\right) \mathrm{d} \Omega
\end{array}\right.
$$

Then subproblem (10) can be rewritten as follows

$$
\min _{\mathbf{a}^{n}} \frac{1}{2}\left(\mathbf{a}^{n}\right)^{T} \cdot \mathbf{G}_{1} \cdot \mathbf{a}^{n}-\mathbf{g}_{1}^{T} \cdot \mathbf{a}^{n} \text {, s.t. }\left\|\mathbf{a}^{n}\right\|_{\infty} \leq \eta_{1} .
$$


It is a quadratic optimization problem with boundedness constraint, which can be conveniently solved by traditional optimization methods, e.g., the trust region reflective method [24].

\subsubsection{Solving subproblem (11)}

Subproblem (11) is not a least squares problem and does not have close-form solution. We adopt the Gauss-Newton method [25] to solve it iteratively. In each iteration, we linearize the term in $\int_{\Omega}(\cdot)^{2} \mathrm{~d} \Omega$ of (11) locally and relax (11) to a least squares problem.

Suppose that $\mathbf{b}_{k}^{n-1}$ has been obtained at the last iteration and denote $h\left(\mathbf{b}^{n-1}\right)=O_{m}-u_{m}^{n}-\Delta t$. $\operatorname{inv}^{T}\left(u_{m}^{n}, v_{m}^{n-1}+\Delta t \cdot \mathbf{i n v}^{T}\left(v_{m}^{n-1}, u_{m}^{n-1}\right) \cdot \mathbf{b}^{n-1}\right) \cdot \mathbf{a}^{n}$. Then the linear approximation of $h$ at $\mathbf{b}_{k}^{n-1}$ is

$$
h\left(\mathbf{b}^{n-1}\right)=h\left(\mathbf{b}_{k}^{n-1}\right)+\left(\frac{D h}{D \mathbf{b}_{k}^{n-1}}\right)^{T} \cdot\left(\mathbf{b}^{n-1}-\mathbf{b}_{k}^{n-1}\right),
$$

where $\frac{D h}{D \mathbf{b}_{k}^{n-1}}$ is the Gâteaux derivatives of $h$ with respect to $\mathbf{b}^{n-1}$. It can be computed as follows:

$$
\frac{D h}{D \mathbf{b}^{n-1}}=-(\Delta t)^{2} \cdot \sum_{(p, q) \in \mathcal{P}} \sigma_{p q}\left(v_{m}^{n}\right) \cdot \operatorname{inv}\left(u_{m}^{n-1}, v_{m}^{n-1}\right),
$$

where

$$
\left\{\begin{aligned}
\sigma_{p q}\left(v_{m}^{n}\right) & =\frac{\partial \mathbf{i n v}^{T}\left(u_{m}^{n}, v_{m}^{n}\right)}{\partial\left(v_{m}^{n}\right)_{p q}} \cdot \mathbf{a}^{n}=\sum_{j=0}^{16} a_{j}^{n} \frac{\partial \operatorname{inv}_{j}\left(u_{m}^{n}, v_{m}^{n}\right)}{\partial\left(v_{m}^{n}\right)_{p q}}, \\
\left(v_{m}^{n}\right)_{p q} & =\frac{\partial^{p+q}\left(v_{m}^{n}\right)}{\partial x^{p} \partial y^{q}}
\end{aligned}\right.
$$

and $\mathcal{P}=\{(0,0),(0,1),(1,0),(2,0),(1,1),(0,2)\}$ is the index set for partial differentiation.

Then we obtain a relaxed problem

$$
\min _{\mathbf{b}^{n-1}} \frac{1}{2} \sum_{m=1}^{M} \int_{\Omega}\left[h\left(\mathbf{b}_{k}^{n-1}\right)+\left(\frac{D h}{D \mathbf{b}_{k}^{n-1}}\right)^{T} \cdot\left(\mathbf{b}^{n-1}-\mathbf{b}_{k}^{n-1}\right)\right]^{2} \mathrm{~d} \Omega \text {, s.t. }\left\|\mathbf{b}^{n-1}\right\|_{\infty} \leq \eta_{2} .
$$

Note that $\mathbf{b}^{n-1}$ is independent of $(x, y)$. Denote

$$
\left\{\begin{array}{l}
\mathbf{g}_{2}=\sum_{m=1}^{M} \int_{\Omega} \frac{D h}{D \mathbf{b}_{k}^{n-1}} \cdot\left[h\left(\mathbf{b}_{k}^{n-1}\right)-\left(\frac{D h}{D \mathbf{b}_{k}^{n-1}}\right)^{T} \cdot \mathbf{b}_{k}^{n-1}\right] \mathrm{d} \Omega, \\
\mathbf{G}_{2}=\sum_{m=1}^{M} \int_{\Omega} \frac{D h}{D \mathbf{b}_{k}^{n-1}} \cdot\left(\frac{D h}{D \mathbf{b}_{k}^{n-1}}\right)^{T} \mathrm{~d} \Omega .
\end{array}\right.
$$

Then problem (17) can be rewritten as follows:

$$
\min _{\mathbf{b}^{n-1}} \frac{1}{2}\left(\mathbf{b}^{n-1}\right)^{T} \cdot \mathbf{G}_{2} \cdot \mathbf{b}^{n-1}+\mathbf{g}_{2}^{T} \cdot \mathbf{b}^{n-1}, \quad \text { s.t. } \quad\left\|\mathbf{b}^{n-1}\right\|_{\infty} \leq \eta_{2} .
$$

It is also a quadratic optimization problem with boundedness constraint, which can be conveniently solved by the trust region reflective method [24]. The iteration terminates when the difference between $\mathbf{b}_{k}^{n-1}$ and $\mathbf{b}_{k-1}^{n-1}$ is sufficiently small. 


\section{Discussions}

In this section, we first discuss the complexity of the proposed approach and then make a comparison between FATSA and GDM [13]. Finally, we extend FATSA to handle vector-valued processing problems.

\subsection{Computational complexity}

In this subsection, we discuss the computational complexity of the proposed FATSA. As we set the maximum time $T=N \Delta t$, we can compute an asymptotic worst-case bound for the time complexity. We denote $n_{\mathbf{i n v}}$ as the number of invariants (for gray image $n_{\mathbf{i n v}}=17$, for color image $n_{\mathbf{i n v}}=69$ ) and $|\Omega|$ as the number of pixels in $\Omega$. Problems (13) and (19) are convex quadratic optimization problems for the positive definite $\mathbf{G}_{1}$ and $\mathbf{G}_{2}$, respectively. The cubic time complexity $O\left(n_{\text {inv }}^{3}\right)$ is an asymptotic worst-case bound. For each iteration in Algorithm 2, we need to update $v_{m}^{n}$. So the time complexity is $O(|\Omega|)$. When we have $M$ image training pairs and the maximum number of iteration is $K, O\left(K\left(M|\Omega|+n_{\text {inv }}^{3}\right)\right)$ is the worst-case bound for Algorithm 2. As the terminal time $T=N \Delta t$, the worst-case bound for Algorithm 1 is $O\left(K N\left(M|\Omega|+n_{\text {inv }}^{3}\right)\right)$.

\subsection{Comparison between FATSA and GDM}

In this subsection, we make a comparison between FATSA and GDM [13]. For the training speed, we compare the training time and errors between FATSA and GDM in Subsection 5.5. It can be seen from Table 4 that the training time is greatly reduced by FATSA, and the training errors of FATSA are obviously lower than those of GDM in all datasets. In this case, FATSA is much faster and more effective than GDM. For the simplicity, we have shown that FATSA minimizes (4) at each time step, while GDM minimizes (5) at $t=T$ only. This makes the learning of PDEs much simpler as it is unnecessary to compute the adjoint PDEs for evaluating the Gâteaux derivatives [23] of the objective functional. For the flexibility, when minimizing (4) at each time step, it can fit for more general regulations (e.g., non-smooth regularization) and extra constraints on the linear combination

coefficients. Moreover, we can still run FATSA when $t$ reaches $T$ until the objective function value of (4) does not decrease sufficiently. For GDM, it is impossible to do so because the dimension of coefficients after temporal discretization is fixed. Although there are more time steps, FATSA is still much faster than GDM (see Table 4).

\subsection{Handling vector-valued images}

In practice, images in many vision tasks are often vector-valued, such as RGB color images, multispectral satellite images and multimodal medical images. FATSA can also work for learning PDEs for 
vector-valued images. In this case, the optimization problem at time $t_{n}$ is

$$
\begin{aligned}
\min _{\mathbf{a}^{n}, \mathbf{b}^{n-1}} L^{n+1} & =\frac{1}{2} \sum_{m=1}^{M} \sum_{c=1}^{C} \int_{\Omega}\left[O_{m}^{c}-u_{m}^{c}\left(t_{n+1}\right)\right]^{2} \mathrm{~d} \Omega, \\
\text { s.t. } & \left\|\mathbf{a}_{c}^{n}\right\|_{\infty}<\eta_{1}, c=1, \cdots, C \text {, and }\left\|\mathbf{b}^{n-1}\right\|_{\infty}<\eta_{2},
\end{aligned}
$$

where $\mathbf{u}_{m}=\left\{u_{m}^{c}, c=1, \cdots, C\right\}: \Omega \rightarrow \mathbb{R}^{C}$ are the evolutionary vector-valued images for input image $\mathbf{I}_{m}$ and expected output images $\mathbf{O}_{m}=\left\{O_{m}^{c}, c=1, \cdots, C\right\}: \Omega \rightarrow \mathbb{R}^{C}$. We simply take the luminance of the input image as the initial function of the indicator function $v$. So the PDEs system consists of $C+1$ evolutionary PDEs. Accordingly, there are much more fundamental differential invariants, which are invariant to translation and rotation. The set of such invariants up to second order is

$$
\left\{1, f_{r},\left(\nabla f_{r}\right)^{T} \cdot \nabla f_{s},\left(\nabla f_{r}\right)^{T} \cdot \mathbf{H}_{f_{m}} \cdot \nabla f_{s}, \operatorname{tr}\left(\mathbf{H}_{f_{r}}\right), \operatorname{tr}\left(\mathbf{H}_{f_{r}} \cdot \mathbf{H}_{f_{s}}\right) \mid f_{r}, f_{s}, f_{m} \in\left\{u^{1}, \cdots, u^{C}, v\right\}\right\}
$$

If $C=3$, there are 69 fundamental differential invariants up to the second order. For more details, please refer to $[13,21,22]$. Obviously, $\left\{\mathbf{a}_{c} \mid c=1, \cdots, C\right\}$ are not coupled with each other. So we can still use alternating minimization to solve (20).

\section{Numerical experiments}

In this section, we first give more details on implementation. Then we conduct extensive experiments on various datasets to test the performance of the proposed FATSA. Note that this paper targets on solving the PDE constrained optimal control problem better. So we mainly focus on comparing FATSA with GDM, rather than comparing the learnt PDEs with state-of-the-art methods for each vision task, which has been shown in [13, 21, 22].

\subsection{Implementation}

To compute the spatial derivatives and integrations, we need to do spatial discretization. We use central difference to approximate the derivatives:

$$
\left\{\begin{aligned}
\frac{\partial f}{\partial x} & =\frac{f(x+1)-f(x-1)}{2} \\
\frac{\partial^{2} f}{\partial x^{2}} & =f(x+1)-2 f(x)+f(x-1) .
\end{aligned}\right.
$$

The discrete forms of $\frac{\partial f}{\partial y}, \frac{\partial^{2} f}{\partial y}$ and $\frac{\partial^{2} f}{\partial x \partial y}$ can be defined similarly. In addition, we discretize the integrations as

$$
\int_{\Omega} f(x, y) \mathrm{d} \Omega=\frac{1}{|\Omega|} \sum_{(x, y) \in \Omega} f(x, y),
$$

where $|\Omega|$ is the number of pixels in $\Omega$. 
In the first two experiments, the numbers $M$ of training image pairs are both 60. For GDM, $\lambda_{1}=\lambda_{2}=10^{-7}, T=1$ and $\Delta t=0.05$. For TATSA, $\eta_{1}=\eta_{2}=10$ and $\Delta t=0.05$, but $T$ is not limited to 1. Rather, we run FATSA until the difference between successive objective function value is below $10^{-6}$ or the number of iteration reached 100 (see Algorithm 1).

\subsection{Image deblurring}

We first compare the characteristics of FATSA and GDM [13] with a fundamental vision problem: image deblurring. For this task, we take the Berkeley image database [26] and generate the input images by blurring images using a $5 \times 5$ Gaussian kernel with $\sigma=1$. The original images are used as the expected output images.

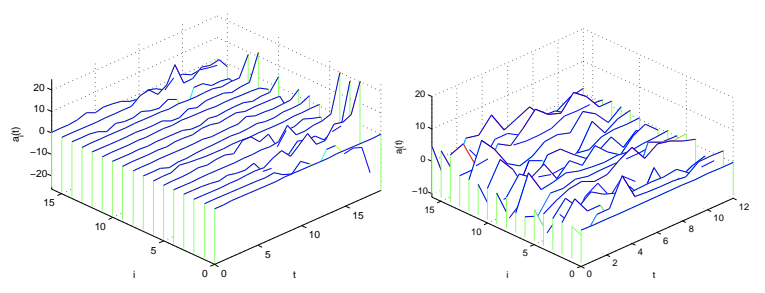

(a) GDM [13]

(b) FATSA

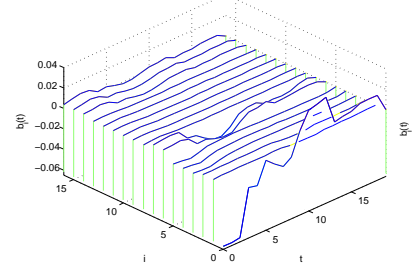

(c) GDM [13]

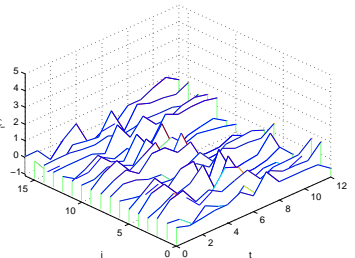

(d) FATSA

Figure 2: The coefficients $a_{i}$ 's $((\mathrm{a}),(\mathrm{b}))$ and $b_{i}$ 's $((\mathrm{c}),(\mathrm{d})), i=0, \cdots, 16$, learnt of GDM [13] and FATSA for debluring.

The coefficients learnt by GDM and FATSA for image debluring are shown in Figure 2. We can observe that the coefficients $a_{i}$ 's learnt by GDM (Figure 2(a)) have a large variance in magnitude and some $a_{i}$ 's increase abruptly to 20 at the last time step. This may cause numerical instability, which we indeed have encountered. In comparison, the coefficients $a_{i}$ 's learnt by FATSA (Figure 4(b)) are much more balanced, which makes the learnt PDEs more numerically stable. The coefficients $b_{i}$ 's learnt by GDM (Figure 4(c)) are very small (at a magnitude of $10^{-2}$ ), which implies that the evolution of indicator function is not effective. In comparison, the magnitudes of coefficients $b_{i}$ 's learnt by FATSA (Figure 4(d)) are much larger, which shows that the indicator function evolves effectively over time.

As for the performance of deblurring, the mean PSNR of FATSA over 200 test images is 32.60dB, while that of GDM is 31.98dB. Some deblurring results are shown in Figure 3. We can see that FATSA restores more edges and textures. It is clear that the images processed by FATSA are sharper than those processed by GDM. 

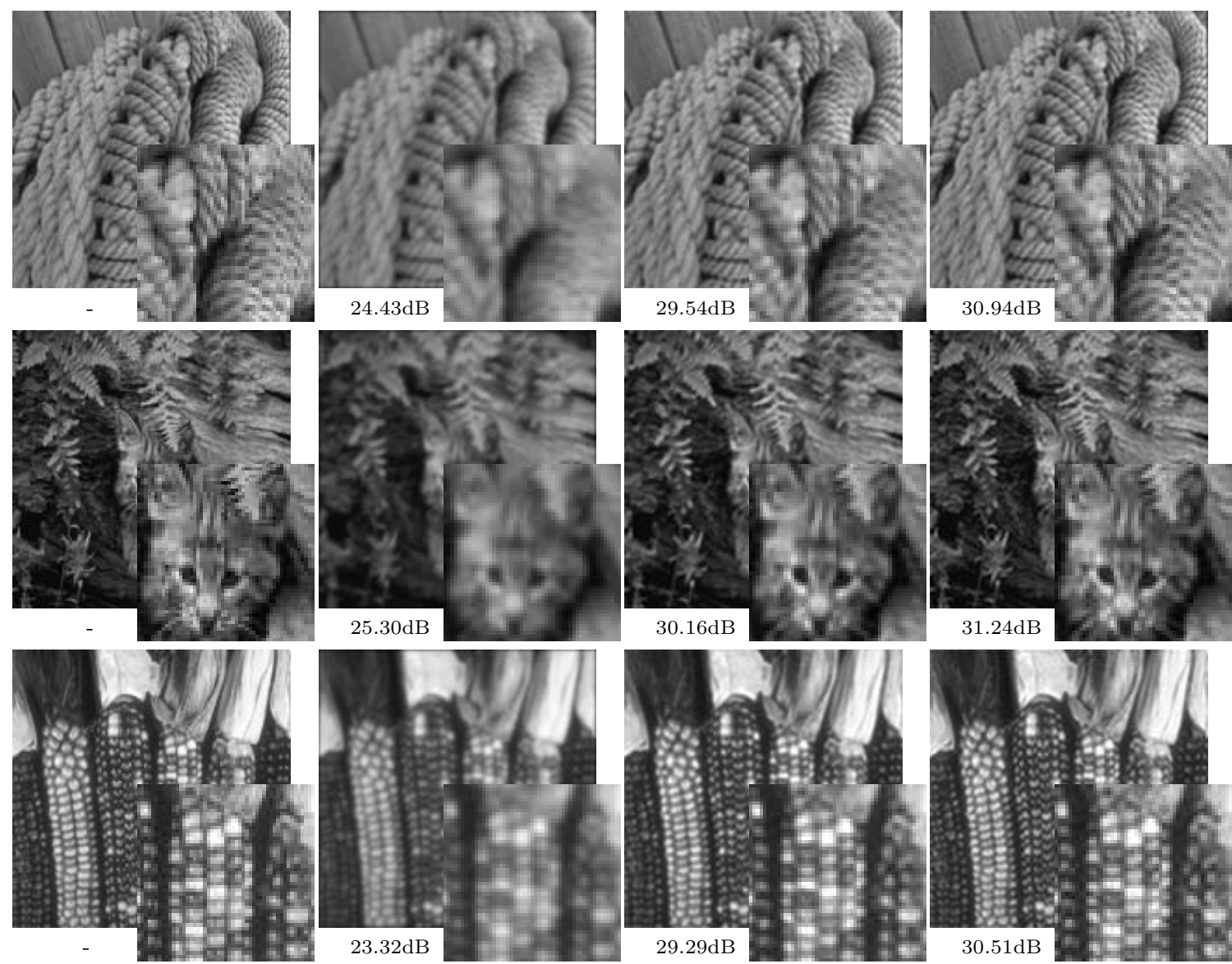

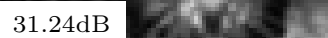

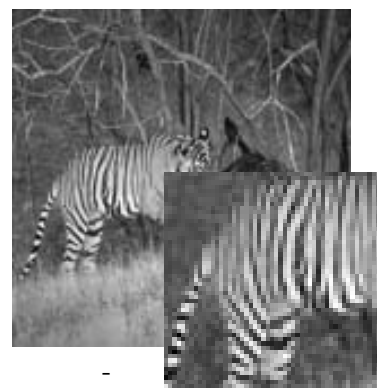

(a)

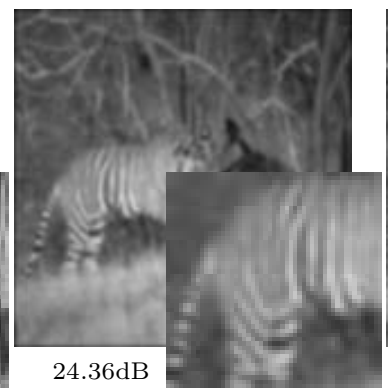

(b)

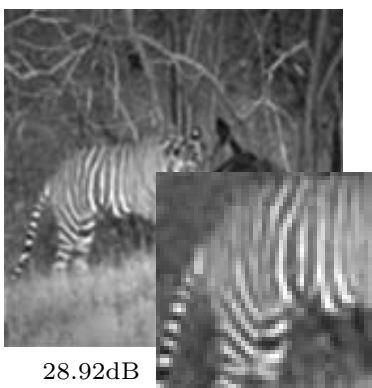

(c)
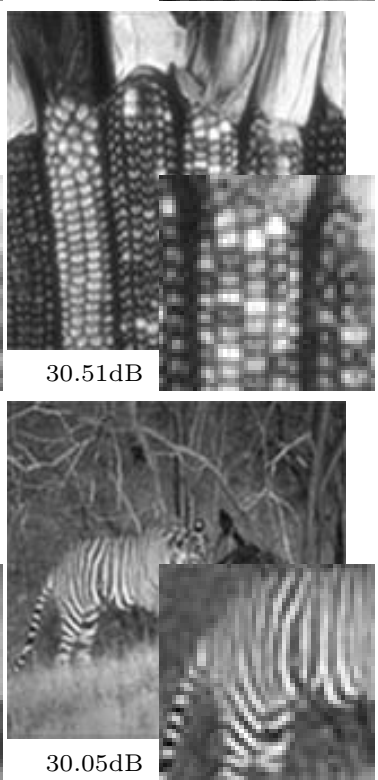

(d)

Figure 3: The results of image deblurring. (a) Ground truth (GT) images. (b) The images blurred by a Gaussian kernel. (c-d) The deblurring results of GDM [13] and FATSA, respectively. The PSNRs are presented below each image.

\subsection{Natural image denoising}

We then apply FATSA to learn PDEs for another fundamental vision problem: image denoising. For this task, we use the images tested in [13] with unknown natural noises. There are 240 images, each with a size of $150 \times 150$ pixels, of 11 objects taken by a Canon 30D digital camera, whose ISO is 1600. For each object, 30 images are taken without changing the camera settings (by fixing the focus, 
aperture and exposure time) and without moving the camera position. The average image of them can be regraded as the ground truth noiseless image, which serve as output training images.

We first compare the coefficients learnt by FATSA and those by GDM [13]. From Figure 4, we can observe the similar phenomenon as in image deblurring. Most of the coefficients $a_{i}$ 's learnt by GDM (Figure 4(a)) are zeros and some jump to more than 20, which may result in numerical instability. The coefficients $a_{i}$ 's learnt by FATSA (Figure 4(b)) are much smoother. Their magnitudes are also much more balanced. Moreover, the coefficients $b_{i}$ 's learnt by GDM (Figure 4(c)) are very small (at the scale of $10^{-4}$ ), which implies that the indicator function actually does not change much over time. In comparison, the magnitudes of coefficients $b_{i}$ 's learnt by FATSA (Figure $4(\mathrm{~d})$ ) are much larger.

Next, we compare the denoising results on the test images between GDM [13] and FATSA. Some results are given in Figure 5. We can see that both GDM and FATSA can remove noise effectively, but FATSA gives the sharper results. Moreover, the PSNRs of FATSA are higher than those of GDM.

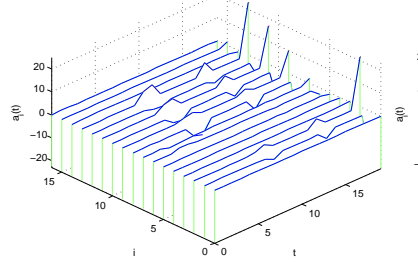

(a) GDM [13]

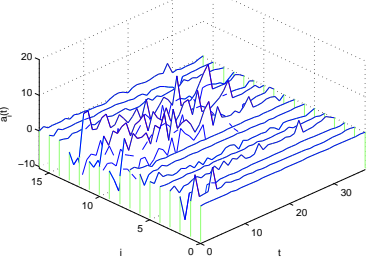

(b) FATSA

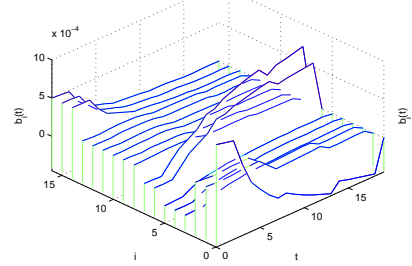

(c) GDM [13]

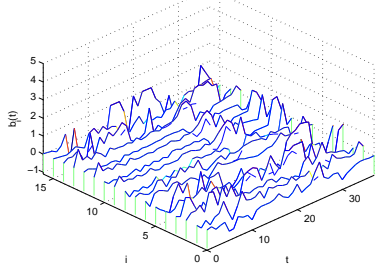

(d) FATSA

Figure 4: The coefficients $a_{i}$ 's $((\mathrm{a}),(\mathrm{b}))$ and $b_{i}$ 's $((\mathrm{c}),(\mathrm{d})), i=0, \cdots, 16$, learnt by GDM [13] and FATSA in image denoising.

\subsection{Color image demosaicking}

In this subsection, we consider a color image processing problem: image demosaicking. Demosaicking is to infer the two missing color components for every pixel from a raw image, which only captures one color component at every pixel due to the filtering of color filter arrays (CFAs) [27, 28]. The most commonly used CFA is the Bayer CFA. For demosaicking, we test our method on both clean and noisy images. For the clean images demosaicking, we choose the Kodak image database [29]. For the noisy images demosaicking, we choose the publicly available noisy dataset [30].

\subsubsection{Demosaicking on the clean images}

First, we take the Kodak image database [29] for clean images demosaicking. Images $1 \sim 12$ are used for training and images $13 \sim 24$ are used for testing. As for saving time and memory cost of training, we divide each $512 \times 768$ image into 12 non-overlapping $150 \times 150$ patches and choose the first 

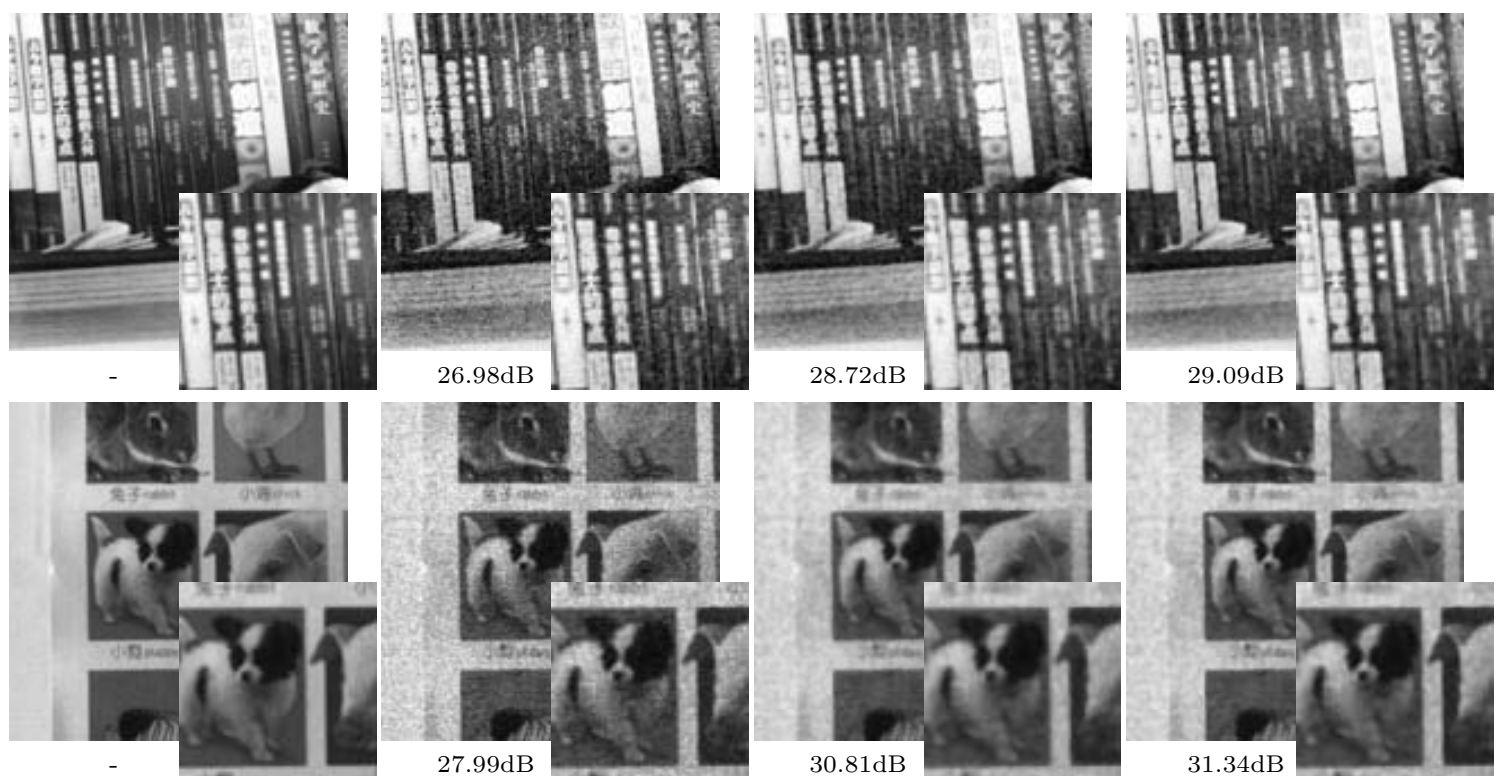

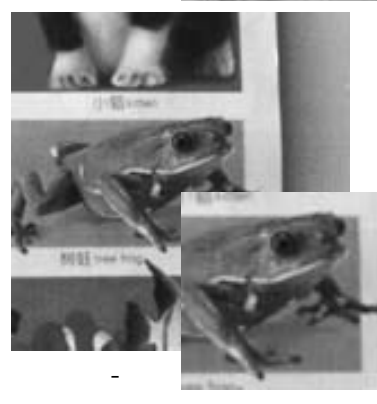

(a)

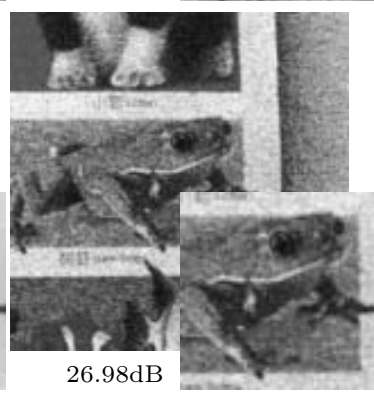

(b)

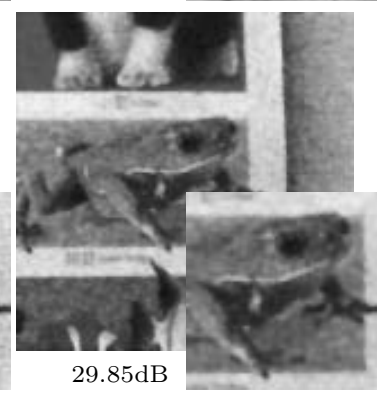

(c)

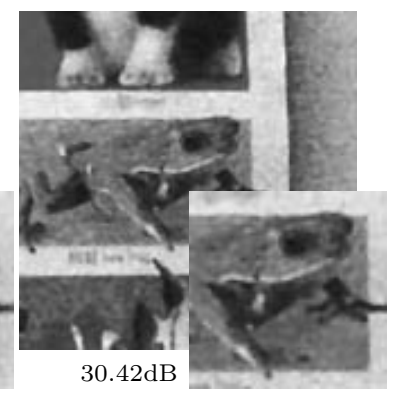

(d)

Figure 5: The results of denoising images with natural noise. (a) Ground truth (GT) images. (b) Images with natural noise. (c-d) Denoised images using GDM [13] and the proposed FATSA, respectively. The PSNRs are presented below each image.

60 patches with the richest texture, which is measured by their variances. After that we downsample the patches into Bayer CFA raw image. Then we bilinearly interpolate the CFA raw data (i.e., for each channel the missing values are bilinearly interpolated from their nearest four available values) into full-color images and use them as the input images of the training pairs. Note that bilinear interpolation (BI) is the naivest way of inferring the missing colors and many artifacts can occur. We use the original full color clean images as the output images of the training pairs. We compare the results of FATSA with those of GDM [13] on the test images.

Figure 6 shows some demosaciking results. We can see that FATSA is better than GDM in all PSNR value, noise reduction, and color fidelity. GDM [13] gives blurry results with many color artifacts, while FATSA is capable of eliminating most of the noises. Table 2 shows the comparison on the mean PSNRs 

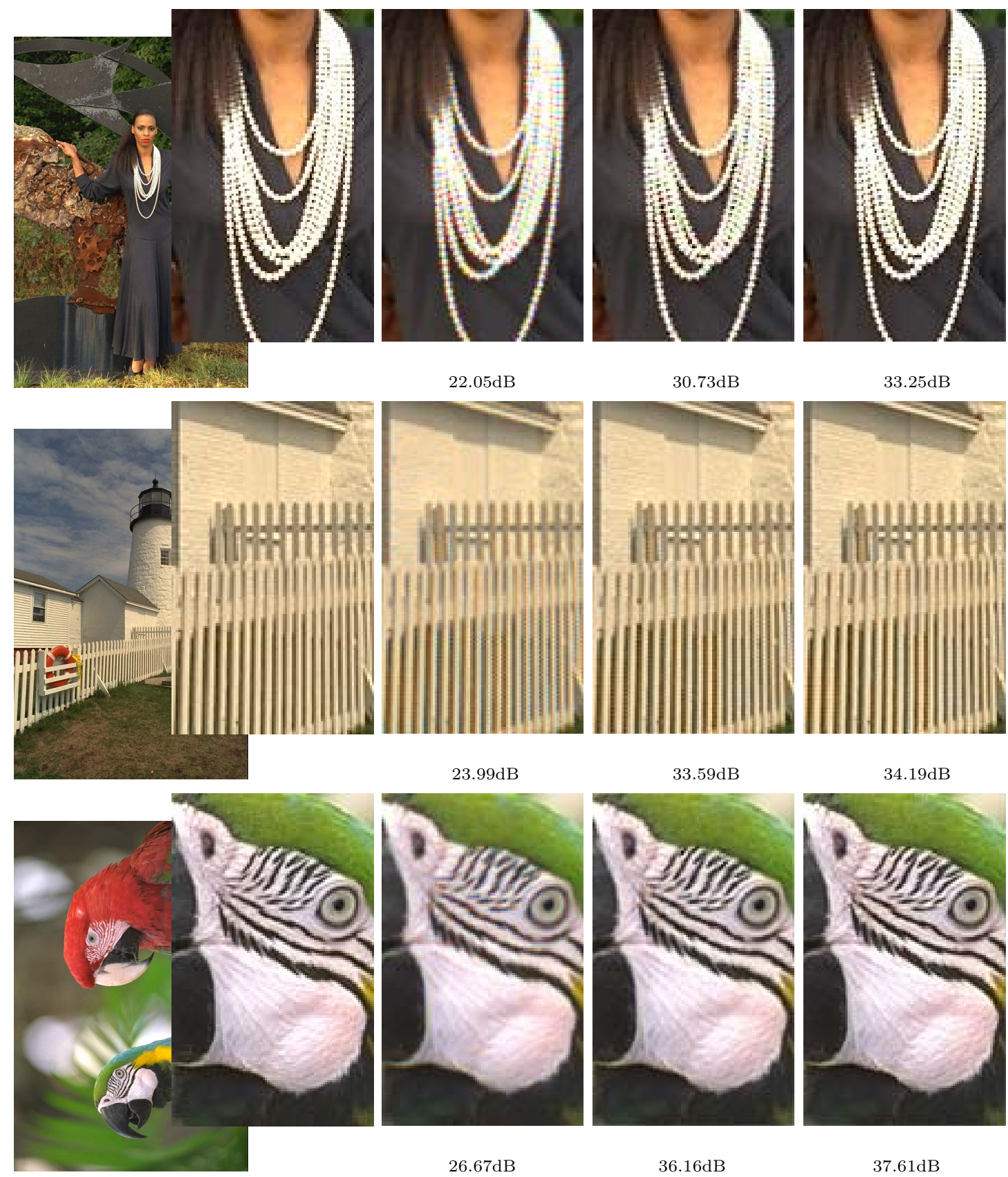

(a)

(b)

(c)

(d)

Figure 6: The demosaicking results for clean images. (a) Ground truth (GT) images. (b-d) The demosaicking results of bilinearly interpolation (BI), GDM [13] and FATSA, respectively. The PSNRs are presented below each image.

of 12 test images. It can be seen that the mean PSNRs of FATSA are much higher than those of GDM. 


\subsubsection{Demosaicking on the noisy images}

Next, we want to show that FATSA can also work very well for noisy images demosaicking. For better comparison, we choose the publicly available Image Denoising Benchmark dataset [30], which is generated from the Berkeley Segmentation Database and images are degraded by additive, uncorrelated Gaussian noise with standard deviations (std) of 5, 10, 15, 25 and 35. We divide each $320 \times 480$ image into 6 non-overlapping $150 \times 150$ patches and select the first 60 patches with the richest texture, which is measured by their variances. Then we downsample the patches into Bayer CFA raw data and use bilinearly interpolated results as the input images of the training pairs.

Some results are given in Figure 7. It shows the demosaicking results under different noise levels. We can see that the proposed FATSA can be used to fully recover Bayer CFA images with low noise levels and it preserves more texture information than GDM [13]. It is clear that the proposed FATSA gives sharper images and less color artifacts in high noise levels. Also, we compute the mean PSNRs on this dataset and show them in Table 3. We can see that the proposed FATSA performs better than GDM [13] in all noise levels.

\subsection{Comparison of training error and time with GDM}

Finally, we compare the training error and training time between GDM [13] and FATSA. The training error is measured by (5), where $M=60$ for image deblurring and noising and $M=12$ for image demosaicking. The Matlab implementation of GDM and FATSA are run on a PC equipped with a dual $3.4 \mathrm{GHz} \mathrm{CPU}$ and $8 \mathrm{~GB}$ memory.

The training error and time of GDM and FATSA are shown in Table 4. Obviously, the training time are greatly reduced by FATSA. For grayscale images, the training speed is accelerated by ten times. For color images, the training time is cut by more than half. Moreover, the tranining errors of FATSA are lower than those of GDM. So we can conclude that FATSA is much more effective than GDM on learning PDEs.

\section{Conclusion}

In this paper, we propose a new fast alternating time-splitting approach (FATSA) to efficiently learn PDEs for different visual tasks. We aim at minimizing the difference between the expected

Table 2: Comparison on the mean PSNRs on 12 test images in the Kodak image database.

\begin{tabular}{ccc}
\hline Input (BI) & GDM [13] & FATSA \\
\hline $31.42 \pm 5.58 \mathrm{~dB}$ & $37.96 \pm 3.75 \mathrm{~dB}$ & $\mathbf{3 8 . 7 1} \pm 3.61 \mathrm{~dB}$ \\
\hline
\end{tabular}


Table 3: Comparison on the mean PSNRs for increasing noise level of different algorithms.

\begin{tabular}{cccc} 
Noise (std) & Input (BI) & GDM [13] & FATSA \\
\hline 5 & $28.63 \mathrm{~dB}$ & $31.46 \mathrm{~dB}$ & $\mathbf{3 1 . 6 0 d B}$ \\
10 & $27.64 \mathrm{~dB}$ & $29.37 \mathrm{~dB}$ & $\mathbf{2 9 . 3 9} \mathrm{dB}$ \\
15 & $24.47 \mathrm{~dB}$ & $27.88 \mathrm{~dB}$ & $\mathbf{2 7 . 9 2} \mathrm{dB}$ \\
25 & $20.39 \mathrm{~dB}$ & $25.93 \mathrm{~dB}$ & $\mathbf{2 6 . 0 1} \mathrm{dB}$ \\
35 & $17.73 \mathrm{~dB}$ & $24.52 \mathrm{~dB}$ & $\mathbf{2 4 . 8 3} \mathrm{dB}$ \\
\hline
\end{tabular}

Table 4: Comparison of traning error and time of GDM and FATSA

\begin{tabular}{c|cc|cc}
\hline \multirow{2}{*}{} & \multicolumn{2}{|c|}{ Error } & \multicolumn{2}{c}{ Time (s) } \\
\cline { 2 - 5 } & GDM [13] & FATSA & GDM [13] & FATSA \\
\hline Debluring & 1516 & $\mathbf{1 1 8 0}$ & 4769 & $\mathbf{4 9 1}$ \\
Denoising & 1602 & $\mathbf{1 5 2 1}$ & 4729 & $\mathbf{4 7 3}$ \\
\hline Demosaicking, clean & 2630 & $\mathbf{2 5 4 8}$ & 13349 & $\mathbf{6 2 1 0}$ \\
Noisy, std=5 & 4827 & $\mathbf{4 6 4 9}$ & 10212 & $\mathbf{2 8 6 9}$ \\
std=10 & 7147 & $\mathbf{7 0 3 5}$ & 11333 & $\mathbf{3 4 9 9}$ \\
std=15 & 9843 & $\mathbf{9 5 3 9}$ & 10293 & $\mathbf{3 7 5 9}$ \\
std=25 & 15056 & $\mathbf{1 4 9 2 3}$ & 13325 & $\mathbf{5 3 3 6}$ \\
std=35 & 21087 & $\mathbf{2 0 2 6 1}$ & 12263 & $\mathbf{5 0 2 1}$ \\
\hline
\end{tabular}

output and the actual output of PDEs at every time step, rather than at the final step only as GDM does. FATSA is much faster than GDM and also much simpler as it does not require deducing and solving the adjoint equations. Moreover, it is more flexible than GDM in incorporating more complex regularizations or constraints on the linear combination coefficients. Experiments on typical image processing problems show that FATSA outperforms GDM.

In the future, we plan to improve and enrich our work in the following aspects. First, we want to carry out theoretical analysis on the convergence behavior of FATSA and the properties of learnt PDEs. Second, we would like to combine other regularizations and constraints to improve the performance of learnt PDEs. Finally, beyond learning-based PDEs, we will apply FATSA to solve other PDE constrained optimal control problems in computer vision [20], such as optical flow estimation [12, 31], tracking [32, 33] and image sequence interpolation models [34, 35]. 


\section{Acknowledgment}

Zhenyu Zhao is supported by NSF China (grant nos. 61473302). Zhouchen Lin is supported by 973 Program of China (grant no. 2015CB3525), NSF China (grant nos. 61272341 and 61231002), and MSRA. The authors thank Jianlong Wu for helping on revising and proofreading the paper.

\section{References}

[1] Gilles Aubert and Pierre Kornprobst. Mathematical problems in image processing: partial differential equations and the calculus of variations, volume 147. Springer, 2006.

[2] Vicent Caselles, Jean-Michel Morel, Guillermo Sapiro, and Allen R Tannenbaum. Introduction to the special issue on partial differential equations and geometry-driven diffusion in image processing and analysis. IEEE Transactions on Image Processing, 6(3):269-273, 1998.

[3] Guillermo Sapiro. Geometric partial differential equations and image analysis. Cambridge university press, 2006.

[4] Pietro Perona and Jitendra Malik. Scale-space and edge detection using anisotropic diffusion. IEEE Transactions on Pattern Analysis and Machine Intelligence, 12(7):629-639, 1990.

[5] Min Yang, Jingkun Liang, Jianhai Zhang, Haidong Gao, Fanyong Meng, Li Xingdong, and SungJin Song. Non-local means theory based Perona-Malik model for image denosing. Neurocomputing, 120:262-267, 2013.

[6] Stanley Osher and Leonid I Rudin. Feature-oriented image enhancement using shock filters. SIAM Journal on Numerical Analysis, 27(4):919-940, 1990.

[7] Faming Fang, Guixu Zhang, Fang Li, and Chaomin Shen. Framelet based pan-sharpening via a variational method. Neurocomputing, 129:362-377, 2014.

[8] Marcelo Bertalmio, Guillermo Sapiro, Vincent Caselles, and Coloma Ballester. Image inpainting. In SIGGRAPH, pages 417-424, 2000.

[9] Chunming Li, Chenyang Xu, Changfeng Gui, and Martin D Fox. Level set evolution without re-initialization: a new variational formulation. In CVPR, volume 1, pages 430-436, 2005.

[10] Xuchu Wang, Jinxiao Shan, Yanmin Niu, Liwen Tan, and Shao-Xiang Zhang. Enhanced distance regularization for re-initialization free level set evolution with application to image segmentation. Neurocomputing, 141:223-235, 2014. 
[11] Steven S. Beauchemin and John L. Barron. The computation of optical flow. ACM Computing Surveys (CSUR), 27(3):433-466, 1995.

[12] Qiao Cai, Yafeng Yin, and Hong Man. DSPM: Dynamic structure preserving map for action recognition. In ICME, pages 1-6. IEEE, 2013.

[13] Risheng Liu, Zhouchen Lin, Wei Zhang, Kewei Tang, and Zhixun Su. Toward designing intelligent PDEs for computer vision: An optimal control approach. Image and Vision Computing, 31:43-56, 2013.

[14] Burger Martin, C.G. Mennucci Andrea, Osher Stanley, and Rumpf Martin. Level Set and PDE Based Reconstruction Methods in Imaging. Springer, 2013.

[15] Leonid I Rudin, Stanley Osher, and Emad Fatemi. Nonlinear total variation based noise removal algorithms. Physica D: Nonlinear Phenomena, 60(1):259-268, 1992.

[16] Tony F Chan and Selim Esedoglu. Aspects of total variation regularized $L^{1}$ function approximation. SIAM Journal on Applied Mathematics, 65(5):1817-1837, 2005.

[17] Nir Sochen, Ron Kimmel, and Ravi Malladi. A general framework for low level vision. IEEE Transactions on Image Processing, 7(3):310-318, 1998.

[18] M Ehrhardt and Simon R Arridge. Vector-valued image processing by parallel level sets. IEEE Transactions on Image Processing, 23(1):9-18, 2014.

[19] Risheng Liu, Zhouchen Lin, Wei Zhang, and Zhixun Su. Learning PDEs for image restoration via optimal control. In $E C C V, 2010$.

[20] Kimia Benjamin, Tannenbaum Allen, and Zucker Steven. On optimal control methods in computer vision and image processing. Geometry-Driven Diffusion in Computer Vision, 1:307-338, 1994.

[21] Zhouchen Lin, Wei Zhang, and Xiaoou Tang. Learning partial differential equations for computer vision. Technical report, Technical report, Microsoft Research, MSR-TR-2008-189, 2008.

[22] Zhouchen Lin, Wei Zhang, and Xiaoou Tang. Designing partial differential equations for image processing by combining differential invariants. Technical report, Technical report, Microsoft Research, MSR-TR-2009-192, 2009.

[23] E Zeidler. Nonlinear Functional Analysis and its Applications. Springer-Verlag, 1985. 
[24] Thomas F Coleman and Yuying Li. A reflective Newton method for minimizing a quadratic function subject to bounds on some of the variables. SIAM Journal on Optimization, 6(4):10401058, 1996.

[25] Ake Björck. Numerical methods for least squares problems. SIAM, 1996.

[26] P Arbelaez, C Fowlkes, and D Martin. The Berkeley segmentation dataset [Online]. Available: http://www. eecs . berkeley.edu/Research/Projects/CS/vision/bsds/, 2013.

[27] B.K. Gunturk, Y. Altunbasak, and R.M. Mersereau. Color plane interpolation using alternating projections. IEEE Transactions on Image Processing, 11(9):997 - 1013, 2002.

[28] B. Leung, G. Jeon, and E. Dubois. Least-squares lumachroma demultiplexing algorithm for Bayer demosaicking. IEEE Transactions on Image Processing, 20(7):1885-1894, 2011.

[29] True color Kodak images [Online]. Available: http://rOk.us/graphics/kodak/, 2013.

[30] F. J. Estrada. Image denoising benchmark [online]. Available: http://www.cs.utoronto.ca/ strider/Denoise/Benchmark/, 2010.

[31] Nicolas Papadakis, Thomas Corpetti, and Etienne Mémin. Dynamically consistent optical flow estimation. In $I C C V, 2007$.

[32] Nicolas Papadakis and Etienne Mémin. Variational optimal control technique for the tracking of deformable objects. In ICCV, 2007.

[33] Qi Wang, Jianwu Fang, and Yuan Yuan. Multi-cue based tracking. Neurocomputing, 131:227-236, 2014.

[34] Kanglin Chen and Dirk A Lorenz. Image sequence interpolation using optimal control. Journal of Mathematical Imaging and Vision, 41(3):222-238, 2011.

[35] Kanglin Chen and Dirk A Lorenz. Image sequence interpolation based on optical flow, segmentation, and optimal control. IEEE Transactions on Image Processing, 21(3):1020-1030, 2012. 

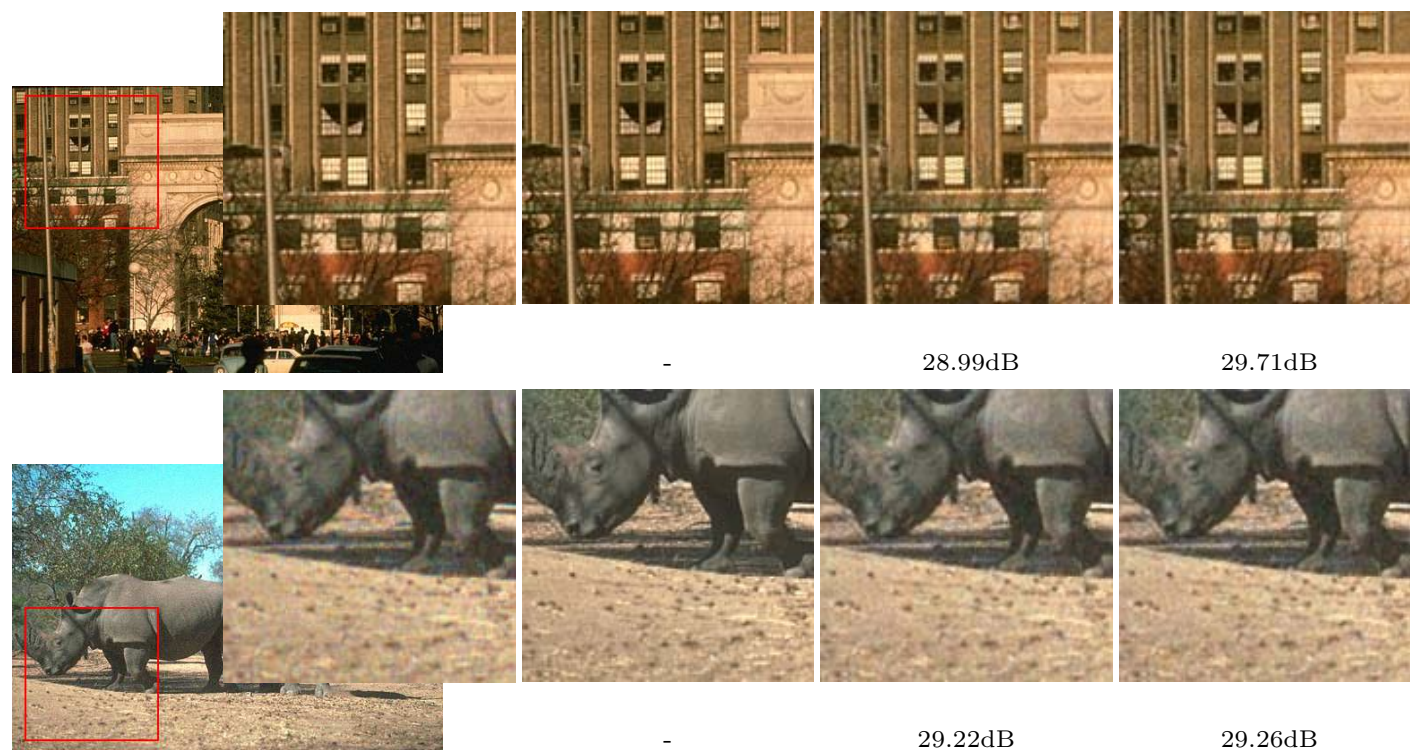

28.99 dB

$29.71 \mathrm{~dB}$
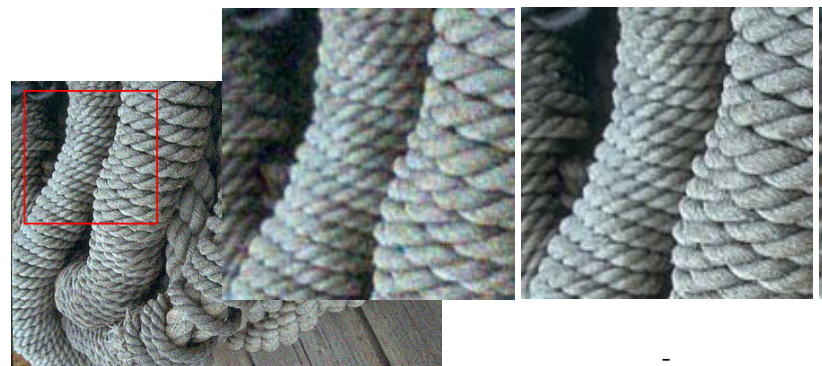

$29.22 \mathrm{~dB}$

$29.26 \mathrm{~dB}$
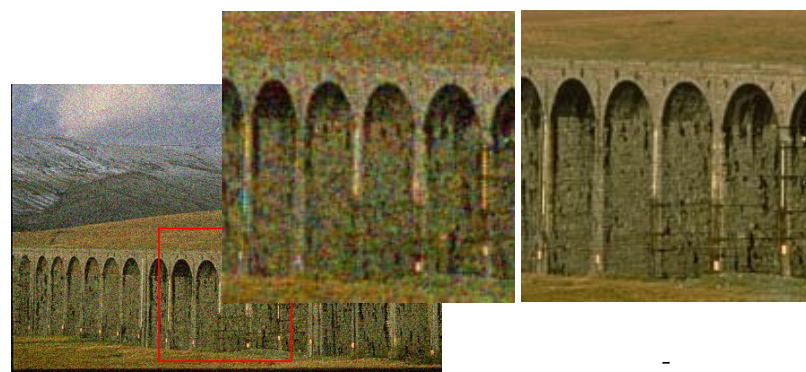

$28.96 \mathrm{~dB}$
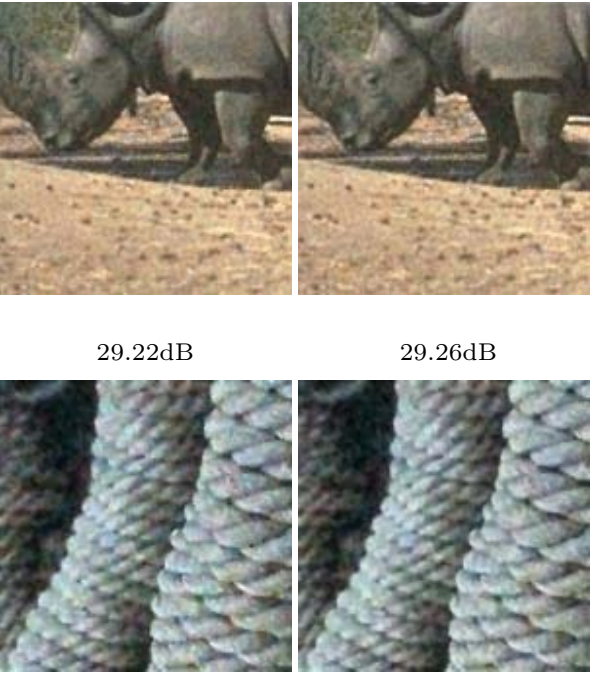

$29.13 \mathrm{~dB}$
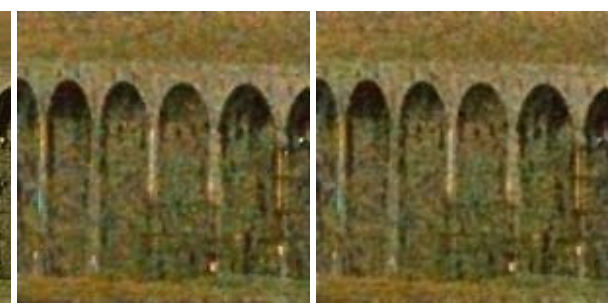

$24.97 \mathrm{~dB}$

$25.11 \mathrm{~dB}$
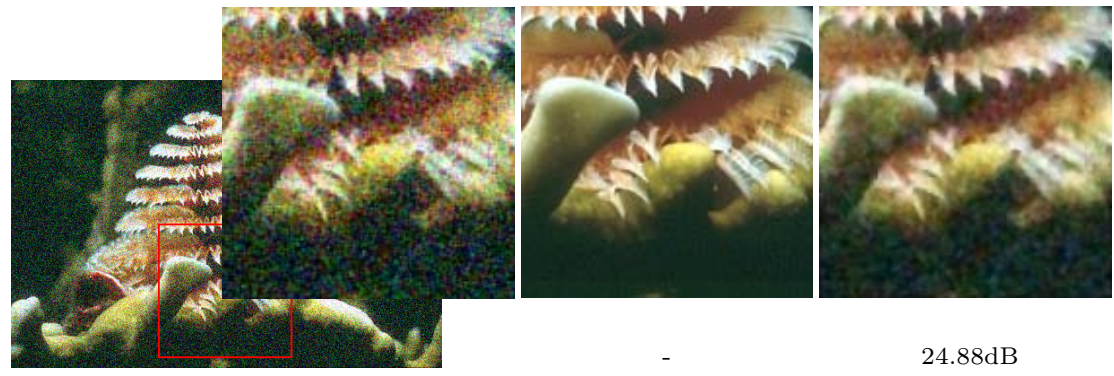

$24.88 \mathrm{~dB}$

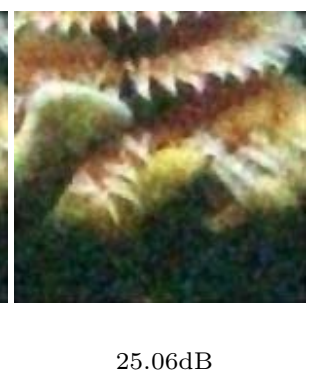

(c)

(d)

Figure 7: The demosaicking results for noisy images (From the first row to the last row, images are degraded by Gaussian noise with standard deviations (std) of 5, 10, 15, 25 and 35, respectively). (a) Noisy images with different noise level. (b) Ground truth (GT) images. (c-d) The demosaicking results of GDM [13] and the proposed FATSA. 

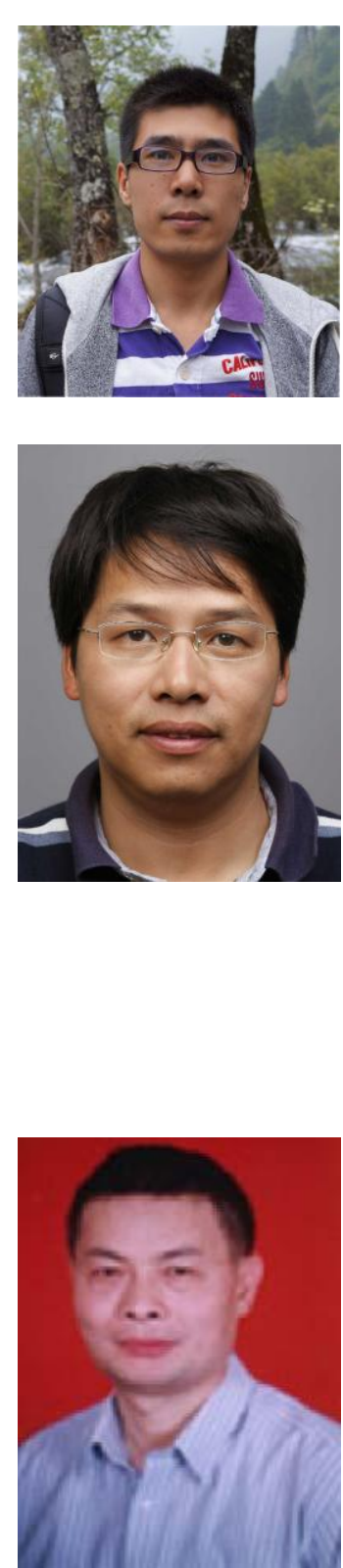

Zhenyu Zhao received the B.S. degree in mathematics from University of Science and Technology in 2009, and the M.S. degree in system science from National University of Defense and Technology in 2011. He is currently pursuing the Ph.D. degree in applied mathematics, National University of Defense and Technology. His research interests include computer vision, pattern recognition and machine learning.

Zhouchen Lin received the PhD degree in applied mathematics from Peking University in 2000. Currently, he is a professor at the Key Laboratory of Machine Perception (MOE), School of Electronics Engineering and Computer Science, Peking University. He is also a chair professor at Northeast Normal University. He was a guest professor at Shanghai Jiaotong University, Beijing Jiaotong University, and Southeast University. He was also a guest researcher at the Institute of Computing Technology, Chinese Academic of Sciences. His research interests include computer vision, image processing, machine learning, pattern recognition, and numerical optimization. $\mathrm{He}$ is an associate editor of IEEE T. Pattern Analysis and Machine Intelligence and International J. Computer Vision and a senior member of the IEEE.

$\mathrm{Yi} \mathbf{W u}$ is a professor in the Department of Mathematics and System Science at the National University of Defense Technology in Changsha, China. He earned bachelor's and master's degrees in applied mathematics at the National University of Defense Technology in 1981 and 1988. He worked as a visiting researcher at NewYork State University in 1999.His research interests include applied mathematics, statistics, and data processing. 
*Photo of the author(s) Click here to download Photo c

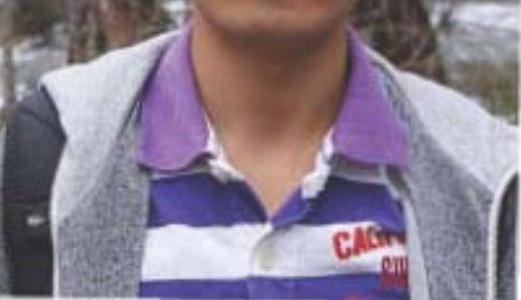




\section{${ }^{*}$ Photo of the-author(s)}
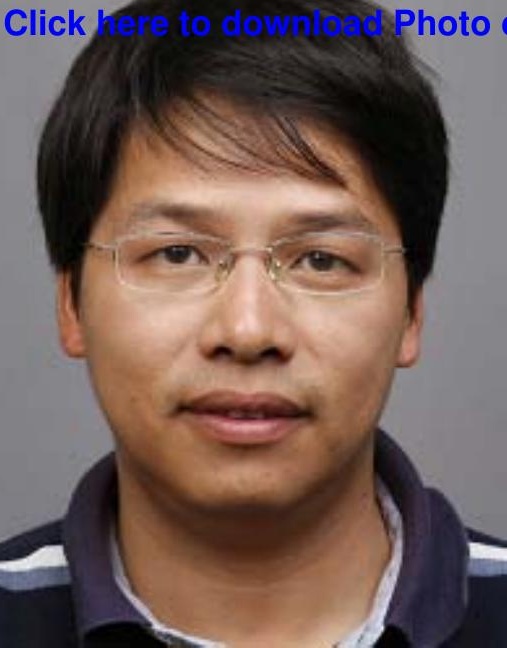


\section{*Photo of the author(s)}
Click here
lad
Photo

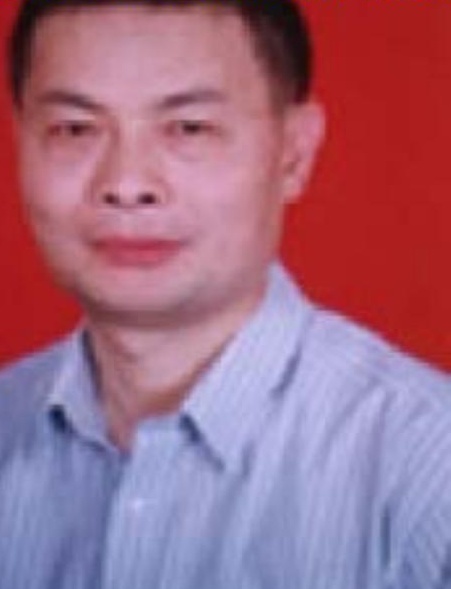

